\title{
ON LINEAR PRESERVERS OF SEMIPOSITIVE MATRICES*
}

\author{
SACHINDRANATH JAYARAMAN ${ }^{\dagger}$ AND VATSALKUMAR N. MER VAR $^{\ddagger}$
}

\begin{abstract}
Given proper cones $K_{1}$ and $K_{2}$ in $\mathbb{R}^{n}$ and $\mathbb{R}^{m}$, respectively, an $m \times n$ matrix $A$ with real entries is said to be semipositive if there exists a $x \in K_{1}^{\circ}$ such that $A x \in K_{2}^{\circ}$, where $K^{\circ}$ denotes the interior of a proper cone $K$. This set is denoted by $S\left(K_{1}, K_{2}\right)$. We resolve a recent conjecture on the structure of into linear preservers of $S\left(\mathbb{R}_{+}^{n}, \mathbb{R}_{+}^{m}\right)$. We also determine linear preservers of the set $S\left(K_{1}, K_{2}\right)$ for arbitrary proper cones $K_{1}$ and $K_{2}$. Preservers of the subclass of those elements of $S\left(K_{1}, K_{2}\right)$ with a $\left(K_{2}, K_{1}\right)$-nonnegative left inverse as well as connections between strong linear preservers of $S\left(K_{1}, K_{2}\right)$ with other linear preserver problems are considered.
\end{abstract}

Key words. Semipositive matrices, Row positive matrices, Inverse nonnegative matrices, Linear preserver problems, Rank one preserver, Proper cones.

AMS subject classifications. 15A86, 15B48.

1. Introduction. We work throughout over the field $\mathbb{R}$ of real numbers. Let $M_{m, n}$ denote the set of all $m \times n$ matrices over $\mathbb{R}$. When $m=n$, this set will be denoted by $M_{m}$ or $M_{n}$. A matrix $A \in M_{m, n}$ is said to be semipositive if there exists a $x>0$ such that $A x>0$, where the inequalities are understood componentwise. $A$ is said to be minimally semipositive if it is semipositive and no proper $m \times p$ submatrix of $A$ is semipositive for $p<n$. $A$ is said to be redundantly semipositive if it is semipositive but not minimally semipositive. It is known that an $m \times n$ matrix $A$ is minimally semipositive if and only if $A$ is semipositive and $A$ has a nonnegative left inverse. Semipositivity characterizes invertible M-matrices within the class of Z-matrices (see Chapter 6, [4]). For recent results on semipositive matrices, their structure and preservers, one may refer to $[1,7,9,19]$ and the references cited therein.

For a field $\mathbb{F}$ and the set $M_{m, n}(\mathbb{F})$ of $m \times n$ matrices over $\mathbb{F}$, a linear preserver $L$ is a linear map $L: M_{m, n}(\mathbb{F}) \longrightarrow M_{m, n}(\mathbb{F})$ that preserves a certain property or a relation. There are two types of preserver problems. Given a subset $\mathcal{S}$ of $M_{m, n}(\mathbb{F})$, characterize linear maps $L$ on $M_{m, n}(\mathbb{F})$ such that $(i) L(\mathcal{S}) \subset$ $\mathcal{S}$ and $($ ii $) L(\mathcal{S})=\mathcal{S}$. The first one is called an into preserver and the latter an onto/strong preserver. There is rich history on this topic within linear algebra as well as other areas of mathematics.

Recall that an $m \times n$ matrix $A$ is row positive if $A$ is a nonnegative matrix with at least one nonzero entry in each row. A square matrix $B$ is said to be a monomial matrix if in addition to being a nonnegative matrix, each row and column of $B$ contains exactly one nonzero entry. The starting point and motivation for this work comes from the following results (Theorems 2.4, 2.11 and Corollary 2.7) due to Dorsey et al. [9].

1. (Theorem 2.4, [9]) Let $L(A)=X A Y$ for some $X \in M_{m}$ and $Y \in M_{n}$. Then $L$ is an into preserver of semipositivity if and only if $X$ is row positive and $Y$ is inverse nonnegative, or $-X$ is row positive

\footnotetext{
* Received by the editors on April 29, 2020. Accepted for publication on December 4, 2020. Handling Editor: Michael Tsatsomeros. Corresponding Author: Sachindranath Jayaraman.

${ }^{\dagger}$ School of Mathematics, Indian Institute of Science Education and Research Thiruvananthapuram, Maruthamala P.O., Vithura, Thiruvananthapuram - 695 551, Kerala, India (sachindranathj@gmail.com, sachindranathj@iisertvm.ac.in)

${ }^{\ddagger}$ SQC \& OR Unit, Indian Statistical Institute, 7, S. J. S Sansanwal Marg, New Delhi - 110016, India (vnm232657@gmail.com, vatsal.n15@iisertvm.ac.in)
} 
and $-Y$ is inverse nonnegative. $L$ is an onto preserver of semipositivity if and only if $X$ and $Y$ are monomial, or $-X$ and $-Y$ are monomial (Corollary 2.7, [9]).

2. (Theorem 2.11, [9]) Let $L(A)=X A Y$ for some $X \in M_{m}$ and $Y \in M_{n}$. Then $L$ is an into preserver of minimal semipositivity if and only if $X$ is monomial and $Y$ is inverse nonnegative, or $-X$ is monomial and $-Y$ is inverse nonnegative. $L$ is an onto preserver of minimal semipositivity if and only if $X$ and $Y$ are monomial, or $-X$ and $-Y$ are monomial.

Our primary aim in this manuscript is to resolve the following conjecture due to Dorsey et al. [9]:

Conjecture 1.1. Let $L: M_{m, n} \rightarrow M_{m, n}$ be an invertible linear map. If $L$ is an into preserver of $S\left(\mathbb{R}_{+}^{n}, \mathbb{R}_{+}^{m}\right)$, then $L(A)=X A Y$ for all $A \in M_{m, n}$, where $X$ is row positive and $Y$ is inverse nonnegative.

Dorsey et al. had pointed out through an example that invertibility of the map $L$ is crucial in the above conjecture (see the example in Section 4 of [9]). They had also added that there was computational evidence that the conjecture is true in the $2 \times 2$ case, but had no proof nor a counterexample.

The purpose of this manuscript is twofold. The first one concerns resolving Conjecture 1.1. The second part concerns linear preservers of the set $S\left(K_{1}, K_{2}\right)$ (see the next section for definitions and notations). We begin by recalling preliminaries about convex sets, nonnegative, and semipositive matrices. Other necessary results on convex cones and positive operators are presented in a later section when we discuss linear preservers of $S\left(K_{1}, K_{2}\right)$. Our first result says that if an invertible linear map $L$ preserves the set of semipositive matrices and also maps every rank one semipositive matrix to a rank one (semipositive) matrix, then $L$ is a rank one preserver (Theorem 3.1) and consequently, $L(A)=X A Y$ for invertible matrices $X$ and $Y$. This result provides a hint for resolving Conjecture 1.1. Our main result says that an invertible linear map $L$ on $M_{m, n}$ that preserves semipositive matrices is of the form $L(A)=X A Y$ for all $A \in M_{m, n}$ for some invertible row positive matrix $X \in M_{m}$ and inverse nonnegative matrix $Y \in M_{n}$ if and only if every rank one semipositive matrix of a special form gets mapped to a rank one matrix (see Theorem 3.12). The structure of invertible maps $L$ on $M_{2}$ that preserve semipositivity (Theorems 3.3) is discussed following Theorem 3.1. A similar argument also works for maps on $M_{m, 2}$. The proof is constructive and we deduce that $L(A)=X A Y$ for some row positive matrix $X$ and inverse nonnegative $Y$. The general case is taken up next. The proof follows ideas that were verified for an invertible linear map $L$ on $M_{3}$ (and more generally on $M_{m, 3}, m \geq 3$ ) that preserves semipositive matrices. Since the calculations are involved and lengthy, the details of the $3 \times 3$ case are not included in the main part of the manuscript. We include these as an Appendix. The second part of the manuscript concerns linear preservers of the set $S\left(K_{1}, K_{2}\right)$. This section is subdivided into further subsections as follows: (1) useful results, (2) linear preservers of $S\left(K_{1}, K_{2}\right),(3)$ preservers of $M S\left(K_{1}, K_{2}\right)$ (see the next section for the definition), (4) general strong preservers of $S\left(K_{1}, K_{2}\right)$, and (5) left semipositivity and their preservers. Interesting connections between onto preservers of $S\left(K_{1}, K_{2}\right)$ and into preservers of nonnegativity are also brought out. To the best of our knowledge this appears new and completely settles this problem both over all proper cones, including the nonnegative orthants.

REMARK 1.2. We shall use the same notation for a linear map $T: \mathbb{R}^{n} \rightarrow \mathbb{R}^{m}$ and its matrix representation, which we assume throughout to be with respect to the standard basis of $\mathbb{R}^{n}$ and $\mathbb{R}^{m}$, respectively.

2. Preliminary results. We present the preliminary results in this section.

2.1. Convex cones, nonnegative, and semipositive matrices. Let us recall that a subset $K$ of $\mathbb{R}^{n}$ is called a convex cone if $K+K \subseteq K$ and $\alpha K \subseteq K$ for all $\alpha \geq 0$. $K$ is said to be proper if it is topologically closed, pointed $(K \cap-K=\{0\})$, and has nonempty interior $K^{\circ} . K$ is said to be polyhedral 
if $K=X\left(\mathbb{R}_{+}^{m}\right)$ for some $n \times m$ matrix $X$ and simplicial when $X$ is invertible. The dual, $K^{*}$, is defined as $K^{*}=\left\{y \in \mathbb{R}^{n}:\langle y, x\rangle \geq 0 \forall x \in K\right\}$, where $\langle.,$.$\rangle denotes the usual Euclidean inner product on \mathbb{R}^{n}$. When $K$ is a convex cone in $\mathbb{R}^{n}$ such that $K=K^{*}$, we say that $K$ is a self-dual cone in $\mathbb{R}^{n}$. The most well-known example of a proper (convex) self-dual cone is the nonnegative orthant in $\mathbb{R}^{n}: K=\mathbb{R}_{+}^{n}=\{x=$ $\left.\left(x_{1}, \ldots, x_{n}\right)^{t} \in \mathbb{R}^{n}: x_{i} \geq 0 \forall 1 \leq i \leq n\right\}$. We assume that all cones in this manuscript are proper cones.

The following definitions, notations, and basic results are standard (see, for instance, [3]). The only exception is Definition 2.2, which is a natural generalization to proper cones the notion of minimally semipositive matrices (see [12] for the definition).

DeFinition 2.1. For proper cones $K_{1}$ and $K_{2}$ in $\mathbb{R}^{n}$ and $\mathbb{R}^{m}$, respectively, we have the following notions. $A \in M_{m, n}$ is

1. $\left(K_{1}, K_{2}\right)$-nonnegative if $A\left(K_{1}\right) \subseteq K_{2}$.

2. $\left(K_{1}, K_{2}\right)$-semipositive if there exists a $x \in K_{1}^{\circ}$ such that $A x \in K_{2}^{\circ}$.

We denote the set of all matrices that are $\left(K_{1}, K_{2}\right)$-nonnegative by $\pi\left(K_{1}, K_{2}\right)$. When $K_{1}=K_{2}=K$, this will be denoted by $\pi(K)$. Let us also denote the set of all matrices that are $\left(K_{1}, K_{2}\right)$-semipositive by $S\left(K_{1}, K_{2}\right)$. When $K_{1}=K_{2}=K$, this will be denoted by $S(K)$.

Definition 2.2. Let $A \in M_{m, n}$ be $\left(K_{1}, K_{2}\right)$-semipositive. We say $A$ is $\left(K_{1}, K_{2}\right)$-minimally semipositive if $A$ has a $\left(K_{2}, K_{1}\right)$-nonnegative left inverse.

The set of all $\left(K_{1}, K_{2}\right)$-minimally semipositive matrices will be denoted by $M S\left(K_{1}, K_{2}\right)$.

Definition 2.3. A square matrix $Y$ is $K$-inverse nonnegative if $Y$ is invertible with $Y^{-1}$ being $K$ nonnegative.

3. Main results. The main results of this manuscript are presented in this section. As stated previously, our aim in this manuscript is to resolve Conjecture 1.1. The primary motivation for this problem comes from Theorems 2.4, 2.11, and 3.5 due to Dorsey et al. [9]. We assume that $m \geq n$ and $n \geq 2$.

3.1. The nonnegative orthants case. We begin by proving that if $L$ is an invertible linear preserver of semipositive matrices that also maps every rank one semipositive matrix to a rank one (semipositive) matrix, then $L$ is in the standard form. It is obvious that if $L(A)=X A Y$ for some invertible row positive matrix $X$ and inverse nonnegative matrix $Y$, the map $L$ has the property mentioned above.

3.1.1. A sufficient condition. For $0 \neq x \in \mathbb{R}^{m}$ and consider the set $U_{x}:=\left\{x u^{t}: u \in \mathbb{R}^{n}\right\}$, an $n$-dimensional subspace of $M_{m, n}$ consisting of matrices rank at most one.

TheOREM 3.1. Let $L: M_{m, n} \rightarrow M_{m, n}$ be an invertible linear map. Assume that L satisfies the following conditions:

1. $L$ is an into preserver of $S\left(\mathbb{R}_{+}^{n}, \mathbb{R}_{+}^{m}\right)$.

2. $\operatorname{rank}(L(A))=1$, whenever $\operatorname{rank}(A)=1$ and $A \in S\left(\mathbb{R}_{+}^{n}, \mathbb{R}_{+}^{m}\right)$.

Then $L(A)=X A Y$ for any $A \in M_{m, n}$, where $X \in M_{m}$ is invertible and row positive and $Y \in M_{n}$ is inverse nonnegative. 
Proof. The proof involves four steps.

Claim 1: For any $x \in\left(\mathbb{R}_{+}^{m}\right)^{\circ} \cup\left(-\left(\mathbb{R}_{+}^{m}\right)^{\circ}\right)$, any $0 \neq A \in L\left(U_{x}\right)$ has rank one.

Proof: Let $x \in\left(\mathbb{R}_{+}^{m}\right)^{\circ}$. We discuss two cases here.

Case 1: Suppose $u \in \mathbb{R}^{n} \backslash\left(-\mathbb{R}_{+}^{n}\right)$. Then $x u^{t} \in S\left(\mathbb{R}_{+}^{n}, \mathbb{R}_{+}^{m}\right)$ and so $\operatorname{rank}\left(L\left(x u^{t}\right)\right)=1$.

Case 2: $0 \neq u \in-\mathbb{R}_{+}^{n}$. In this case, $x(-u)^{t} \in S\left(\mathbb{R}_{+}^{n}, \mathbb{R}_{+}^{m}\right)$, and once again $\operatorname{rank}\left(L\left(x(-u)^{t}\right)\right)=1$. A similar conclusion holds for $x \in\left(-\mathbb{R}_{+}^{m}\right)^{\circ}$.

Claim 2: For $x \in\left(\mathbb{R}_{+}^{m}\right)^{\circ} \cup\left(-\left(\mathbb{R}_{+}^{m}\right)^{\circ}\right), L\left(U_{x}\right)=U_{y}$ for some $y \in \mathbb{R}^{m}$.

Proof: Suppose the claim is not true. Without loss of generality, assume that there exist linearly independent vectors $u_{1}, u_{2} \in \mathbb{R}^{n}$ such that $x u_{1}^{t}, x u_{2}^{t} \in S\left(\mathbb{R}_{+}^{n}, \mathbb{R}_{+}^{m}\right), L\left(x u_{1}^{t}\right)=z_{1} q_{1}^{t}$ and $L\left(x u_{2}^{t}\right)=z_{2} q_{2}^{t}$, where $z_{1}, z_{2} \in \mathbb{R}_{+}^{n}$ are linearly independent and $q_{1}, q_{2} \in \mathbb{R}^{m}$. We then have $L\left(x\left(u_{1}+u_{2}\right)^{t}\right)=L\left(x u_{1}^{t}\right)+L\left(x u_{2}^{t}\right)=z_{1} q_{1}^{t}+z_{2} q_{2}^{t}$. Since $\operatorname{rank}\left(x\left(u_{1}+u_{2}\right)^{t}\right)=1$, we have $L\left(x\left(u_{1}+u_{2}\right)^{t}\right)=z_{3} q_{3}^{t}$. Therefore, $q_{1}$ and $q_{2}$ must be linearly dependent. We thus have a 2-dimensional subspace of $M_{m, n}$ that is mapped to a 1-dimensional subspace of $M_{m, n}$. This contradiction proves the claim.

Claim 3: For $0 \neq z \in \mathbb{R}^{m}, L\left(U_{z}\right)=U_{p}$ for $0 \neq p \in \mathbb{R}^{m}$.

Proof: If $z \in\left(\mathbb{R}_{+}^{m}\right)^{\circ} \cup\left(-\left(\mathbb{R}_{+}^{m}\right)^{\circ}\right)$, then we have $L\left(U_{z}\right)=U_{p}$, for some $p \in \mathbb{R}^{m}$ (by Claim 2 ). If $z$ belongs to the complement of $\left(\mathbb{R}_{+}^{m}\right)^{\circ} \cup\left(-\left(\mathbb{R}_{+}^{m}\right)^{\circ}\right)$, then we can write $z=z_{1}-z_{2}$, for some linearly independent $z_{1}, z_{2} \in\left(\mathbb{R}_{+}^{m}\right)^{\circ}$. Since $z_{1}$ and $z_{2}$ are linearly independent, there exist linearly independent $p_{1}$ and $p_{2}$ such that $L\left(U_{z_{1}}\right)=U_{p_{1}}$ and $L\left(U_{z_{2}}\right)=U_{p_{2}}$. Suppose there exists $q \in \mathbb{R}^{n}$ such that $L\left(z_{1} q^{t}\right)=p_{1} q_{1}^{t}$ and $L\left(z_{2} q^{t}\right)=p_{2} q_{2}^{t}$, where $q_{1}$ and $q_{2}$ are linearly independent. We then have $L\left(\left(z_{1}+z_{2}\right) q^{t}\right)=p_{1} q_{1}^{t}+p_{2} q_{2}^{t}$, a rank two matrix. This contradiction (using Claim 1) proves that $L\left(U_{z}\right)=U_{p}$, for some $0 \neq p \in \mathbb{R}^{m}$.

We have thus proved that $L$ preserves the set of rank 1 matrices in $M_{m, n}$. It now follows from Theorem 2 of [13] that $L(A)=X A Y$ or $m=n$ and $L(A)=X A^{t} Y$, for all $A \in M_{m, n}$, for invertible matrices $X$ and $Y$. Since $A \mapsto A^{t}$ need not preserve semipositivity, there exist no invertible matrices $X$ and $Y$ such that the map $A \mapsto X A^{t} Y$ preserves semipositivity. Therefore, the map $A \mapsto X A^{t} Y$ can be ruled out. Finally, Theorem 2.4 of [9] yields the desired conclusion on $X$ and $Y$.

Theorem 2 of [13] is actually a real version of the Marcus-Moyls result on rank one preservers (see Theorem 1 and the Corollary following it in [15]). The idea behind Theorem 3.1 comes from [18]. It gives us a sufficient condition to check for a map to preserve semipositivity. Note that Theorem 3.1 also holds for $m<n$. We prove that if $L$ is an invertible linear map on $M_{m, n}$ that preserves semipositivity, then any rank one semipositive matrix of the form $A_{i}=\mathbf{x y}_{i}^{t}$, where $\mathbf{x}=\left(x_{1}, x_{2}, \ldots, x_{m}\right)^{t} \in \mathbb{R}^{m}$, and $\mathbf{y}_{i}=$ $\left(0, \ldots, y_{i}, \ldots, 0\right)^{t} \in \mathbb{R}^{n}$, gets mapped to a rank one matrix. We exploit this to prove the result for $n=2$.

3.1.2. The $\boldsymbol{n}=\mathbf{2}$ case. Let us observe that a $2 \times 2$ matrix $A$ is semipositive if and only if $A$ has a positive column or has one of the forms

$$
A=\left[\begin{array}{rr}
a & -b \\
-c & d
\end{array}\right] \text { or } A=\left[\begin{array}{rr}
-b & a \\
d & -c
\end{array}\right],
$$

where $a>0, d>0, b \geq 0, c \geq 0$ and $a d-b c>0$. 
Let us take the usual basis $\left\{E_{i j}: i=1,2, j=1,2\right\}$ of $M_{2}$. For a linear map $L$ on $M_{2}$, let us write down the matrix representation of $L\left(E_{i j}\right)$. It is then easy to write the matrix representation of any rank one matrix $A=\mathbf{x y}^{t}$. We do this below.

Let $L$ be a linear map on $M_{2}$ and let $A=\mathbf{x y}^{t}$ be a rank one matrix, where $\mathbf{x}=\left(x_{1}, x_{2}\right)^{t}$ and $\mathbf{y}=\left(y_{1}, y_{2}\right)^{t}$. We then have

$$
L(A)=\left[\begin{array}{rr}
\left(\alpha_{1} x_{1}+\alpha_{3} x_{2}\right) y_{1}+\left(\alpha_{2} x_{1}+\alpha_{4} x_{2}\right) y_{2} & \left(\beta_{1} x_{1}+\beta_{3} x_{2}\right) y_{1}+\left(\beta_{2} x_{1}+\beta_{4} x_{2}\right) y_{2} \\
\left(\gamma_{1} x_{1}+\gamma_{3} x_{2}\right) y_{1}+\left(\gamma_{2} x_{1}+\gamma_{4} x_{2}\right) y_{2} & \left(\delta_{1} x_{1}+\delta_{3} x_{2}\right) y_{1}+\left(\delta_{2} x_{1}+\delta_{4} x_{2}\right) y_{2}
\end{array}\right]
$$

where $\alpha_{i}, \beta_{i}, \gamma_{i}, \delta_{i}, i=1, \ldots, 4$ are fixed real numbers. In other words, we have

$L(A)=\left[\begin{array}{rr}\left(\alpha_{1} x_{1}+\alpha_{3} x_{2}\right) & \left(\beta_{1} x_{1}+\beta_{3} x_{2}\right) \\ \left(\gamma_{1} x_{1}+\gamma_{3} x_{2}\right) & \left(\delta_{1} x_{1}+\delta_{3} x_{2}\right)\end{array}\right] y_{1}+\left[\begin{array}{rr}\left(\alpha_{2} x_{1}+\alpha_{4} x_{2}\right) & \left(\beta_{2} x_{1}+\beta_{4} x_{2}\right) \\ \left(\gamma_{2} x_{1}+\gamma_{4} x_{2}\right) & \left(\delta_{2} x_{1}+\delta_{4} x_{2}\right)\end{array}\right] y_{2}$.

A similar form exists for $n \geq 3$ that will be used later.

THEOREM 3.2. Let $L$ be an invertible linear map on $M_{2}$ and $L\left(S\left(\mathbb{R}_{+}^{2}\right)\right) \subset S\left(\mathbb{R}_{+}^{2}\right)$. If $A_{1}=\boldsymbol{x} \boldsymbol{y}_{1}^{t} \in S\left(\mathbb{R}_{+}^{2}\right)$ and $A_{2}=\boldsymbol{x} \boldsymbol{y}_{2}^{t} \in S\left(\mathbb{R}_{+}^{2}\right)$, where $\boldsymbol{x}=\left(x_{1}, x_{2}\right)^{t}, \boldsymbol{y}_{1}=\left(y_{1}, 0\right)^{t}$ and $\boldsymbol{y}_{2}=\left(0, y_{2}\right)^{t}$, then rank $\left(L\left(A_{1}\right)\right)=1$ and rank $\left(L\left(A_{2}\right)\right)=1$. Moreover, $L\left(A_{1}\right)=\boldsymbol{u v}^{t} y_{1}$ and $L\left(A_{2}\right)=\boldsymbol{p} \boldsymbol{q}^{t} y_{2}$, where $\boldsymbol{u}=\left(\left(\alpha_{1} x_{1}+\alpha_{3} x_{2}\right),\left(\gamma_{1} x_{1}+\gamma_{3} x_{2}\right)\right)^{t}, \boldsymbol{v}=$ $(1,-\alpha)^{t}, \boldsymbol{p}=\left(\left(\beta_{2} x_{1}+\beta_{4} x_{2}\right),\left(\delta_{2} x_{1}+\delta_{4} x_{2}\right)\right)^{t}, \boldsymbol{q}=(-\gamma, 1)^{t},\left[\begin{array}{ll}\alpha_{1} & \alpha_{3} \\ \gamma_{1} & \gamma_{3}\end{array}\right] \geq 0\left[\begin{array}{cc}\beta_{2} & \beta_{4} \\ \delta_{2} & \delta_{4}\end{array}\right] \geq 0, \alpha \geq 0 \& \gamma \geq 0$

Proof. The proof involves several steps.

Claim 1: $L\left(A_{1}\right)$ and $L\left(A_{2}\right)$ are not minimally semipositive.

Suppose there exists a $A_{1}=\mathbf{x y}_{1}^{t} \in S\left(\mathbb{R}_{+}^{2}\right)$ such that $L\left(A_{1}\right)$ is a minimally semipositive matrix. We know that either $L\left(A_{1}\right)=\left[\begin{array}{rr}a & -b \\ -c & d\end{array}\right] y_{1}$ or $L\left(A_{1}\right)=\left[\begin{array}{rr}-b & a \\ d & -c\end{array}\right] y_{1}$, where $a>0, d>0, b \geq 0, c \geq 0$, and $a d>b c$. Consider the matrix $B=\left[\begin{array}{ll}-x_{1} & x_{1} y_{2} \\ -x_{2} & x_{2} y_{2}\end{array}\right]$, where $y_{2}>0$. It is clear that $B$ is semipositive. If the inverse of $L\left(\left[\begin{array}{ll}-x_{1} & 0 \\ -x_{2} & 0\end{array}\right]\right)$ is negative, it is possible to choose a $y_{2}>0$, sufficiently small, such that the inverse of $L(B)$ is nonpositive. It follows that $L(B) \notin S\left(\mathbb{R}_{+}^{2}\right)$. If the inverse of $L\left(\left[\begin{array}{ll}-x_{1} & 0 \\ -x_{2} & 0\end{array}\right]\right)$ is nonpositive, say $L\left(\left[\begin{array}{ll}-x_{1} & 0 \\ -x_{2} & 0\end{array}\right]\right)=\left[\begin{array}{rr}-a & b \\ 0 & -d\end{array}\right]$ with $a>0, b>0, d>0$, then $L(B)=\left[\begin{array}{rr}-a & b \\ 0 & -d\end{array}\right]+\left[\begin{array}{ll}* & * \\ f & *\end{array}\right]$. If $f \geq 0$, then choose a sufficiently small $y_{2}>0$ such that the inverse of $L(B)$ is nonpositive, thereby making $L(B)$ not semipositive. If $f<0$, then choose $y_{2}>0$ such that the second row of $L(B)$ is negative. This once again makes $L(B)$ not semipositive. This proves the claim. Thus, $L\left(A_{1}\right)$ must be a redundantly semipositive matrix.

Claim 2: $L\left(A_{1}\right)$ cannot have a positive row.

If $L\left(A_{1}\right)$ has positive row, then by choosing $y_{2}>0$ sufficiently small and by taking $B=\left[\begin{array}{ll}-x_{1} & x_{1} y_{2} \\ -x_{2} & x_{2} y_{2}\end{array}\right] \in$ $S\left(\mathbb{R}_{+}^{2}\right)$, we can show that $L(B)$ contains nonpositive row and consequently will not be semipositive. 
We can thus assume without loss of generality that $L\left(A_{1}\right)=\left[\begin{array}{ll}a & -b \\ c & -d\end{array}\right] y_{1}$, where $a>0, c>0, b \geq 0, d \geq 0$. Thus, $L\left(A_{1}\right)=\left[\begin{array}{cc}\alpha_{1} x_{1}+\alpha_{3} x_{2} & \beta_{1} x_{1}+\beta_{3} x_{2} \\ \gamma_{1} x_{1}+\gamma_{3} x_{2} & \delta_{1} x_{1}+\delta_{3} x_{2}\end{array}\right] y_{1}$.

Claim 3: $\left(\alpha_{1}, \alpha_{3}\right)^{t}$ and $\left(\beta_{1}, \beta_{3}\right)^{t}$ are linearly dependent.

Suppose $\left(\alpha_{1}, \alpha_{3}\right)^{t}$ and $\left(\beta_{1}, \beta_{3}\right)^{t}$ are linearly independent. Consider the invertible matrix $W=\left[\begin{array}{cc}\alpha_{1} & \alpha_{3} \\ \beta_{1} & \beta_{3}\end{array}\right]$ and let $d=\left(-d_{1},-d_{2}\right)^{t}<0$. Take $B=\left[\begin{array}{ll}p_{1} & x_{1} y_{2} \\ p_{2} & x_{2} y_{2}\end{array}\right] \in S\left(\mathbb{R}_{+}^{2}\right)$, where $p=\left(p_{1}, p_{2}\right)^{t}$ is such that $W p=d, x_{1}>0$, $x_{2}>0$ and $y_{2}>0$ is sufficiently small enough. It is then possible to make the first row of $L(B)$ is negative, thereby making $L(B)$ not semipositive. This contradiction proves the claim.

Since $\alpha_{1} x_{1}+\alpha_{3} x_{2}=a$ and $\beta_{1} x_{1}+\beta_{3} x_{2}=-b$, there exists $\alpha \geq 0$ such that $\left(\beta_{1}, \beta_{3}\right)^{t}=-\alpha\left(\alpha_{1}, \alpha_{3}\right)^{t}$. Similarly, we can show that $\left(\delta_{1}, \delta_{3}\right)^{t}=-\beta\left(\gamma_{1}, \gamma_{3}\right)^{t}$, where $\beta \geq 0$.

Claim 4: The matrix $\left[\begin{array}{ll}\alpha_{1} & \alpha_{3} \\ \gamma_{1} & \gamma_{3}\end{array}\right] \geq 0$.

If $\left(\alpha_{1}, \alpha_{3}\right)^{t}$ contains both positive and negative entries, then there exists $\left(z_{1}, z_{2}\right)^{t}>0$ with $\left(\alpha_{1}, \alpha_{3}\right)\left(z_{1}, z_{2}\right)^{t}=$ 0 . Then, by taking the semipositive matrix $B=\left[\begin{array}{ll}z_{1} & 0 \\ z_{2} & 0\end{array}\right]$, we see that $L(B)$ contains a zero row. Thus, $L(B) \notin S\left(\mathbb{R}_{+}^{2}\right)$, which implies that $\left(\alpha_{1}, \alpha_{3}\right)^{t} \geq 0$. Similarly, we can show that $\left(\gamma_{1}, \gamma_{3}\right)^{t} \geq 0$.

Claim 5: $\alpha=\beta$.

Suppose $\alpha \neq \beta$. We first consider the $\beta>\alpha$ case; the other case is similar.

Case 1: $\beta-\alpha>0$.

Since $L$ is invertible, the matrix $V=\left[\begin{array}{ll}\alpha_{1} & \alpha_{3} \\ \gamma_{1} & \gamma_{3}\end{array}\right]$ is invertible. Let $\left(q_{1}, q_{2}\right)^{t} \in \mathbb{R}^{2}$ such that $V\left(q_{1}, q_{2}\right)^{t}=(-1,1)^{t}$. Let $B=\left[\begin{array}{ll}q_{1} & x_{1} y_{2} \\ q_{2} & x_{2} y_{2}\end{array}\right] \in S\left(\mathbb{R}_{+}^{2}\right)$, where $x_{1}$ and $x_{2}$ are positive and $y_{2}>0$. As in Claim 1 , we can choose $y_{2}>0$ that is sufficiently small such that $L(B)$ either has a nonpositive inverse or a nonpositive row, thereby making it not semipositive. Thus, this case does not arise.

Case 2: $\beta-\alpha<0$. This can be dealt with similarly as in Case 1.

Hence, we have $L\left(A_{1}\right)=\mathbf{u v}^{t} y_{1}$.

By the previous argument, we can show that either $L\left(A_{2}\right)=\mathbf{j k}^{t} y_{2}$, where $\mathbf{j}=\left(\left(\alpha_{2} x_{1}+\alpha_{4} x_{2}\right),\left(\gamma_{2} x_{1}+\right.\right.$ $\left.\left.\gamma_{4} x_{2}\right)\right)^{t}$ and $\mathbf{k}=(1,-\gamma)^{t}$ or $L\left(A_{2}\right)=\mathbf{p q}^{t} y_{2}$.

Claim 6: $L\left(A_{2}\right)$ cannot be in the form $\mathbf{j} \mathbf{k}^{t} y_{2}$.

Suppose $L\left(A_{2}\right)=\mathbf{j k}^{t} y_{2}$. It can be easily seen that $L\left(A_{1}+A_{2}\right)=\mathbf{u v}^{t} y_{1}+\mathbf{j k}^{t} y_{2}$. As $L$ is invertible, $\left[\begin{array}{r}1 \\ -\alpha\end{array} \quad \begin{array}{r}1 \\ -\gamma\end{array}\right]$ is also invertible. Let us take $\left(-d_{1}-d_{2}\right)^{t}<0$ and discuss two cases. 
Case 1: $-\gamma+\alpha>0$.

There exists $\left(y_{1},-y_{2}\right)^{t} \in \mathbb{R}^{2}$, where $y_{1}$ and $y_{2}$ are positive such that $\left(\alpha_{1} x_{1}+\alpha_{3} x_{2}\right)\left(y_{1}\right)+\left(\alpha_{2} x_{1}+\alpha_{4} x_{2}\right)\left(-y_{2}\right)=$ $-d_{1}$ and $\left(\alpha_{1} x_{1}+\alpha_{3} x_{2}\right)\left(y_{1}\right)(-\alpha)+\left(\alpha_{2} x_{1}+\alpha_{4} x_{2}\right)\left(-y_{2}\right)(-\gamma)=-d_{2}$. Observe now that the first row of $L\left(\left[\begin{array}{ll}y_{1} x_{1} & -y_{2} x_{1} \\ y_{1} x_{2} & -y_{2} x_{2}\end{array}\right]\right)$ is negative.

Case 2: $-\gamma+\alpha<0$.

There exists $\left(-y_{1}, y_{2}\right)^{t} \in \mathbb{R}^{2}$, where $y_{1}$ and $y_{2}$ are positive, such that $\left(\alpha_{1} x_{1}+\alpha_{3} x_{2}\right)\left(-y_{1}\right)+\left(\alpha_{2} x_{1}+\right.$ $\left.\alpha_{4} x_{2}\right)\left(y_{2}\right)=-d_{1}$ and $\left(\alpha_{1} x_{1}+\alpha_{3} x_{2}\right)\left(-y_{1}\right)(-\alpha)+\left(\alpha_{2} x_{1}+\alpha_{4} x_{2}\right)\left(y_{2}\right)(-\gamma)=-d_{2}$. This makes the first row of $L\left(\left[\begin{array}{ll}-y_{1} x_{1} & y_{2} x_{1} \\ -y_{1} x_{2} & y_{2} x_{2}\end{array}\right]\right)$ is negative. Thus, $L\left(A_{2}\right)$ must be in the form $\mathbf{p q}^{t} y_{2}$.

Combining the above claims, the theorem follows.

We now prove our main theorem.

THEOREM 3.3. Let $L$ be an invertible linear map on $M_{2}$. If $L$ is an into preserver of $S\left(\mathbb{R}_{+}^{2}\right)$, then $L(A)=X A Y$, for some invertible row positive $X \in M_{2}$ and an inverse nonnegative $Y \in M_{2}$.

Proof. By Theorem 3.2, we have $L\left(A_{1}\right)=\left[\begin{array}{rr}\left(\alpha_{1} x_{1}+\alpha_{3} x_{2}\right) & -\alpha\left(\alpha_{1} x_{1}+\alpha_{3} x_{2}\right) \\ \left(\gamma_{1} x_{1}+\gamma_{3} x_{2}\right) & -\alpha\left(\gamma_{1} x_{1}+\gamma_{3} x_{2}\right)\end{array}\right] y_{1}$ and $L\left(A_{2}\right)=$ $\left[\begin{array}{lr}-\gamma\left(\beta_{2} x_{1}+\beta_{4} x_{2}\right) & \left(\beta_{2} x_{1}+\beta_{4} x_{2}\right) \\ -\gamma\left(\delta_{2} x_{1}+\delta_{4} x_{2}\right) & \left(\delta_{2} x_{1}+\delta_{4} x_{2}\right)\end{array}\right] y_{2}$, where $\left[\begin{array}{ll}\alpha_{1} & \alpha_{3} \\ \gamma_{1} & \gamma_{3}\end{array}\right]$ and $\left[\begin{array}{cc}\beta_{2} & \beta_{4} \\ \delta_{2} & \delta_{4}\end{array}\right]$ are nonnegative matrices, $\alpha \geq 0$ and $\gamma \geq 0$.

Claim 1: $\left(\alpha_{1}, \alpha_{3}\right)^{t}$ and $\left(\beta_{2}, \beta_{4}\right)^{t}$ are linearly dependent.

Suppose not. We consider two cases. Let $B=\left[\begin{array}{rr}-\alpha_{3} & \beta_{4} \\ \alpha_{1} & -\beta_{2}\end{array}\right]$. If $\operatorname{det}(B)<0$, then $B \in S\left(\mathbb{R}_{+}^{2}\right)$ and the first row of $L(B)$ is zero. If instead $\operatorname{det}(B)>0$, then $-B \in S\left(\mathbb{R}_{+}^{2}\right)$ and the first row of $L(-B)$ is zero. Thus, $\left(\alpha_{1}, \alpha_{3}\right)^{t}$ and $\left(\beta_{2}, \beta_{4}\right)^{t}$ are linearly dependent. This proves the claim.

Similarly, we can show that $\left(\gamma_{1}, \gamma_{3}\right)^{t}$ and $\left(\delta_{2}, \delta_{4}\right)^{t}$ are linearly dependent. Notice that $\left(\beta_{2}, \beta_{4}\right)^{t}=\theta\left(\alpha_{1}, \alpha_{3}\right)^{t}$ and $\left(\delta_{2}, \delta_{4}\right)^{t}=\lambda\left(\gamma_{1}, \gamma_{3}\right)^{t}$, where $\theta>0$ and $\lambda>0$.

Claim 2: $\theta=\lambda$.

Observe that $L\left(\left[\begin{array}{ll}a & b \\ c & d\end{array}\right]\right)=\left[\begin{array}{rr}\alpha_{1} a+\alpha_{3} c-\gamma \theta\left(\alpha_{1} b+\alpha_{3} d\right) & -\alpha\left(\alpha_{1} a+\alpha_{3} c\right)+\theta\left(\alpha_{1} b+\alpha_{3} d\right) \\ \gamma_{1} a+\gamma_{3} c-\gamma \lambda\left(\gamma_{1} b+\gamma_{3} d\right) & -\alpha\left(\gamma_{1} a+\gamma_{3} c\right)+\lambda\left(\gamma_{1} b+\gamma_{3} d\right)\end{array}\right]$.

Suppose $\theta \neq \lambda$. We contradict the $\lambda>\theta$ case; the other case is similar. Say $\lambda>\theta$, and let $d_{1}, d_{2}>0$. We can find $\left(u_{1}, u_{2}\right)^{t} \in \mathbb{R}^{2}$ and $\left(v_{1} v_{2}\right)^{t} \in \mathbb{R}^{2}$ such that $\alpha_{1} u_{1}+\alpha_{3} u_{2}=-d_{1}, \gamma_{1} u_{1}+\gamma_{3} u_{2}=d_{2}, \alpha_{1} v_{1}+\alpha_{3} v_{2}=\frac{d_{1}}{\theta}$ and $\gamma_{1} v_{1}+\gamma_{3} v_{2}=-\frac{d_{2}}{\lambda}$. It can be easily verified that $B=\left[\begin{array}{ll}u_{1} & v_{1} \\ u_{2} & v_{2}\end{array}\right]$ is a minimally semipositive matrix. However, $L(B)=\left[\begin{array}{rr}(1+\gamma)\left(-d_{1}\right) & (1+\alpha) d_{1} \\ (1+\gamma) d_{2} & (1+\alpha)\left(-d_{2}\right)\end{array}\right] \notin S\left(\mathbb{R}_{+}^{2}\right)$. 
Finally, we get $L\left(\left[\begin{array}{ll}a & b \\ c & d\end{array}\right]\right)=\left[\begin{array}{ll}\alpha_{1} & \alpha_{3} \\ \gamma_{1} & \gamma_{3}\end{array}\right]\left[\begin{array}{ll}a & b \\ c & d\end{array}\right]\left[\begin{array}{rr}1 & -\alpha \\ -\gamma \theta & \theta\end{array}\right]$. Observe that $X=\left[\begin{array}{ll}\alpha_{1} & \alpha_{3} \\ \gamma_{1} & \gamma_{3}\end{array}\right]$ is an invertible row positive matrix and $Y=\left[\begin{array}{rr}1 & -\alpha \\ -\gamma \theta & \theta\end{array}\right]$ is inverse nonnegative (see Theorem 2.4, [9]).

REMARK 3.4. Before proceeding further, let us write down the matrix representation of $L\left(A_{i}\right)$ in the general case, where $A_{i}=\mathbf{x y}_{i}^{t}$, with $\mathbf{x}=\left(x_{1}, x_{2}, \ldots, x_{m}\right)^{t} \in \mathbb{R}^{m}$ and $\mathbf{y}_{i}=\left(0, \ldots, y_{i}, \ldots, 0\right)^{t} \in \mathbb{R}^{n}$. Let $L$ be a linear map on $M_{m, n}$. We then have

$$
L\left(A_{i}\right)=\left[\begin{array}{cccc}
s_{1} & s_{2} & \cdots & s_{n} \\
s_{n+1} & s_{n+2} & \cdots & s_{2 n} \\
\vdots & \cdots & \cdots & \vdots \\
s_{(m-1) n+1} & s_{(m-1) n+2} & \cdots & s_{m n}
\end{array}\right] y_{i},
$$

where $s_{k}=l_{k, i} x_{1}+l_{k, n+i} x_{2}+\cdots+l_{k,(m-1) n+i} x_{m}$ and $l_{i, j}, i=1, \ldots, m n, j=1, \ldots, m n$ are fixed real numbers.

The above representation is obtained similar to the $n=2$ case by taking the usual basis $E_{i j}$ of $M_{m, n}$.

REMARK 3.5. Letting $n=2$ in Remark 3.4, we observe that the arguments of Theorems 3.2 and 3.3 carry over for invertible maps on $M_{m, 2}, m \geq 2$ that preserve semipositivity. Recall that when $m \geq 2$, an $m \times 2$ matrix is semipositive if and only if every $2 \times 2$ submatrix is semipositive. We thus have the following theorem.

TheOREM 3.6. Let $L$ be an invertible linear map on $M_{m, 2}$, where $m \geq 2$. Then,

1. $\operatorname{rank}\left(L\left(A_{i}\right)\right)=1$, where $A_{i}$ is as above.

2. $L(A)=X A Y$, for some invertible row positive $X \in M_{m}$ and an inverse nonnegative $Y \in M_{2}$.

3.1.3. The general case: $\boldsymbol{m} \geq \boldsymbol{n}$. We assume that $m \geq n$. As in the $2 \times 2$ case, the first step is to prove that a rank one semipositive matrix of the form $A_{i}$ (as described in REMARK 3.4) does not get mapped to a minimally semipositive matrix. For simplicity, we present a proof in the $3 \times 3$ case, which extends to any $n \times n$ matrix. The reduction to $m \times n$ case follows as an $m \times n$ matrix is semipositive if and only if every $n \times n$ submatrix is semipositive. Recall that a square matrix $A$ is said to be reducible if it is permutation similar to a matrix of the form

$$
\left[\begin{array}{cc}
A_{11} & A_{12} \\
0 & A_{22}
\end{array}\right]
$$

where $A_{11}$ and $A_{22}$ are square and nonzero matrices. $A$ is said to be irreducible if it is not reducible. Two matrices $A$ and $B$ are said to be permutation equivalent if there exist permutation matrices $P$ and $Q$ such that $A=P B Q$. A square matrix $A$ is said to be partially decomposable if it is permutation equivalent to a matrix of the form given above. Otherwise $A$ is said to be fully indecomposable. One can show that $A$ is fully indecomposable if and only if $P A$ is irreducible for every permutation matrix $P$. If $A$ is partially decomposable, then $A$ is permutation equivalent to

$$
\left[\begin{array}{cccc}
A_{11} & A_{12} & \cdots & A_{1 k} \\
0 & A_{22} & \cdots & A_{2 k} \\
\vdots & \cdots & \ddots & \vdots \\
0 & \cdots & 0 & A_{k k}
\end{array}\right]
$$


Electronic Journal of Linear Algebra, ISSN 1081-3810

where the $A_{i i}$ are either $1 \times 1$ zero matrices or are fully indecomposable. We shall use the following notions and results from [11].

- (Corollary 1, [11]) If an $n \times n$ sign pattern matrix $B$ is fully indecomposable, then the following are equivalent.

1. $B$ is inverse nonnegative.

2. $B$ is inverse positive.

- (Theorem 2, [11]) Suppose $B$ is an $n \times n$ decomposable sign pattern matrix in the following block form $B=\left[\begin{array}{cccc}B_{11} & B_{12} & \ldots & B_{1 k} \\ 0 & B_{22} & \ldots & B_{2 k} \\ 0 & \ldots & & B_{3 k} \\ \vdots & \vdots & & \vdots \\ 0 & \ldots & & B_{k k}\end{array}\right]$, where each $B_{i i}$ is square and either fully indecomposable or a $1 \times 1$ zero matrix. Then $B$ is inverse nonnegative if and only if

1. each (fully indecomposable) sign pattern matrix $B_{i i}, i=1, \ldots, k$ is inverse nonnegative.

2. no submatrix of the form $\left[\begin{array}{lll}B_{i, i+1} & \ldots & B_{i j}\end{array}\right]$ or $\left[\begin{array}{c}B_{i j} \\ \vdots \\ B_{j-1, j}\end{array}\right]$ is nonnegative and nonzero, $1 \leq i<$ $j \leq k$.

THEOREM 3.7. Let $L$ be an invertible linear map on $M_{3}$ that preserves semipositive matrices. Consider the rank one matrix $A_{1}$. If $A_{1}$ is semipositive, then $L\left(A_{1}\right)$ cannot be a minimally semipositive matrix.

Proof. Suppose $A_{1}=\left[\begin{array}{l}x_{1} \\ x_{2} \\ x_{3}\end{array}\right]\left[\begin{array}{lll}y_{1} & 0 & 0\end{array}\right]$ is a rank one semipositive matrix, so that $x_{i}>0$ for $i=1,2,3$ and $y_{1}>0$. If $L\left(A_{1}\right)$ is minimally semipositive, then it is inverse nonnegative. We then have the following cases.

Case 1: Suppose $L\left(A_{1}\right)$ is fully indecomposable.

In this case, it follows from Corollary 1, [11] that $L\left(A_{1}\right)$ will have a positive inverse. Consider the semipositive matrix $B=\left[\begin{array}{l}x_{1} \\ x_{2} \\ x_{3}\end{array}\right]\left[\begin{array}{lll}-1 & y_{2} & 0\end{array}\right]$, where $y_{2}>0$. Then $L(B)=L\left(\left[\begin{array}{l}x_{1} \\ x_{2} \\ x_{3}\end{array}\right]\left[\begin{array}{lll}-1 & 0 & 0\end{array}\right]\right)+L\left(\left[\begin{array}{l}x_{1} \\ x_{2} \\ x_{3}\end{array}\right]\left[\begin{array}{lll}0 & y_{2} & 0\end{array}\right]\right)$.

The inverse of the first term is negative, whereas the second term is semipositive. It is now possible to choose a $y_{2}$, sufficiently small, so that the inverse of $L(B)$ is nonpositive. This forces $L(B) \notin S\left(\mathbb{R}_{+}^{3}\right)$.

Case 2: Suppose $L\left(A_{1}\right)$ is partly decomposable and has the form $L\left(A_{1}\right)=\left[\begin{array}{rrr}a & -b & -f_{1} \\ -c & d & -f_{2} \\ 0 & 0 & e\end{array}\right]$, where $a, b, c$, and $d$ are positive, $f_{1}$ and $f_{2}$ are nonnegative and $a d-b c>0$.

Consider the matrix $B$ as in Case 1. Choose $y_{2}>0$ and sufficiently small so that $L(B)=\left[\begin{array}{rrr}-a_{1} & b_{1} & f_{1}^{*} \\ c_{1} & -d_{1} & f_{2}^{*} \\ * & * & -e_{1}\end{array}\right]$, where $a_{1}, b_{1}, c_{1}, d_{1}$ and, $e_{1}$ are positive and $f_{1}^{*}$ and $f_{2}^{*}$ are nonnegative. In this case, it is easy to check that $L(B)$ cannot be minimally semipositive. $L(B)$ cannot be a redundantly semipositive matrix as well, as no $3 \times 2$ submatrix is semipositive. 
Case 3: Suppose $L(A)$ is partly decomposable and has the form $L(A)=\left[\begin{array}{rrr}a & -f_{1} & -f_{2} \\ 0 & d & -f_{3} \\ 0 & 0 & e\end{array}\right]$, where $a$, $d$, and $e$ are positive and $f_{1}, f_{2}$, and $f_{3}$ are nonnegative. This case can be dealt with similar to Case 2 .

Combining everything, we see that $L\left(A_{1}\right)$ cannot be a minimally semipositive matrix.

REMARK 3.8. It follows from the above proof that $L\left(A_{2}\right)$ and $L\left(A_{3}\right)$ cannot be mapped to minimally semipositive matrices as well. Moreover, the above proof works for any $n \geq 4$ with appropriate modifications as well as for the rectangular case.

We have thus proved that no rank one semipositive matrix of the form $A_{i}$ can be mapped to a minimally semipositive matrix. We are now ready to prove our main results for maps on $M_{m, n}$, when $m \geq n \geq 3$.

THEOREM 3.9. Let $A_{1}$ be the rank one matrix described earlier. If $A_{1}$ is semipositive and if $L$ is an invertible linear map on $M_{m, n}$ that preserves semipositivity, then the matrix

$$
C_{1}=\left[\begin{array}{cccc}
l_{1,1} & l_{1,(n+1)} & \ldots & l_{1,(m-1) n+1} \\
l_{2,1} & l_{2,(n+1)} & \ldots & l_{2,(m-1) n+1} \\
\vdots & \vdots & \ldots & \vdots \\
l_{n, 1} & l_{n,(n+1)} & \ldots & l_{n,(m-1) n+1}
\end{array}\right]
$$

has rank one.

Proof. Suppose $C_{1}$ has rank $n$. For $z=-\left(z_{1}, \ldots, z_{n}\right)^{t}<0$, choose a vector $q=\left(q_{1}, \ldots, q_{m}\right)^{t}$ such that $C_{1} q=z$. Consider the matrix $B=\left[\begin{array}{ccccc}q_{1} & x_{1} y_{2} & 0 & \ldots & 0 \\ q_{2} & x_{2} y_{2} & 0 & \ldots & 0 \\ \vdots & \vdots & \vdots & \ldots & \vdots \\ q_{m} & x_{m} y_{2} & 0 & \ldots & 0\end{array}\right]$, where $y_{2}>0$. Then, $B$ is semipositive as it

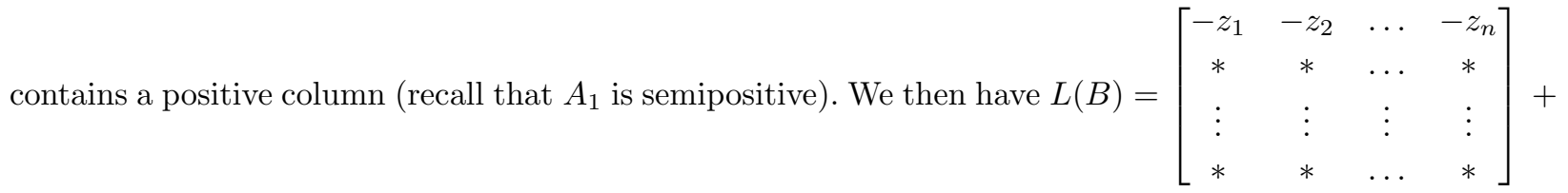
$y_{2} L\left(\left[\begin{array}{ccccc}0 & x_{1} & 0 & \ldots & 0 \\ 0 & x_{2} & 0 & \ldots & 0 \\ \vdots & \vdots & \vdots & \ldots & \vdots \\ 0 & x_{m} & 0 & \ldots & 0\end{array}\right]\right)$. The second term in the above expression is semipositive as $L$ preserves semipositivity. Choosing $y_{2}$ sufficiently small, it is possible to make $L(B)$ not semipositive. Thus, $C_{1}$ cannot have rank $n$.

Consider the matrix $C_{k}=\left[\begin{array}{cccc}l_{1, k} & l_{1,(n+k)} & \ldots & l_{1,(m-1) n+k} \\ l_{2, k} & l_{2,(n+k)} & \ldots & l_{2,(m-1) n+k} \\ \vdots & \vdots & \ldots & \vdots \\ l_{n, k} & l_{n,(n+k)} & \ldots & l_{n,(m-1) n+k}\end{array}\right]$. Let us consider the $n \times m n$ matrix $C=$ $\left[C_{1}\left|C_{2}\right| \ldots \mid C_{n}\right]$. Since $L$ is an invertible map, $C$ has rank $n$. Suppose $C_{1}$ has rank $n-1$. Assume without loss of generality that $\left[C_{1} \mid C_{2}\right]$ has rank $n$. For $z=-\left(z_{1}, \ldots, z_{n}\right)^{t}<0$, choose a $p \in \mathbb{R}^{2 m}$ such that $\left[C_{1} \mid C_{2}\right] p=z$. 
Consider the semipositive matrix $B=\left[\begin{array}{cccccc}p_{1} & p_{m+1} & x_{1} y_{3} & 0 & \ldots & 0 \\ p_{2} & p_{m+2} & x_{2} y_{3} & 0 & \ldots & 0 \\ \vdots & \vdots & \vdots & \vdots & \ldots & \vdots \\ p_{m} & p_{2 m} & x_{m} y_{3} & 0 & \ldots & 0\end{array}\right]$, where $y_{3}>0$. By choosing $y_{3}$ sufficiently small, it is possible to make $L(B)$ not semipositive (the argument is similar to the one used in the previous step). Thus, $C_{1}$ cannot have rank $n-1$. Proceeding analogously, we see that $C_{1}$ cannot have rank $n-2$ and so on, thereby proving that the rank of $C_{1}$ is 1 .

We now prove that if $L$ is an invertible map on $M_{m, n}$ that preserves semipositivity, then $L\left(A_{i}\right)$ has rank one for each $i=1, \ldots, n$.

TheOREM 3.10. Let $L$ be an invertible map on $M_{m, n}$ that preserves semipositivity. Suppose for each $i=1, \ldots, n, A_{i}$ is semipositive. Then, $L\left(A_{i}\right)$ has rank one for each $i=1, \ldots, n$.

Proof. We will prove the result for $A_{1}$. A similar argument works for $i=2, \ldots, n$. We indicate the steps below, where each successive step assumes the previous one. We have already proved that the theorem when $n=2$. Recall that $L\left(A_{1}\right)$ is a redundantly semipositive matrix. The argument presented below has been verified for $n=3$; since the calculations are very lengthy, we are not including them here and a brief explanation is included as an Appendix when $n=3$. Before proceeding further, let us denote by $P_{1}, \ldots, P_{m}$ the following numbers:

- $P_{1}:=l_{1,1} x_{1}+l_{1, n+1} x_{2}+\cdots+l_{1,(m-1) n+1} x_{m}$

- $P_{2}:=l_{n+1,1} x_{1}+l_{n+1, n+1} x_{2}+\cdots+l_{n+1,(m-1) n+1} x_{m}$

- ...

-...

- $P_{m}:=l_{(m-1) n+1,1} x_{1}+l_{(m-1) n+1, n+1} x_{2}+\cdots+l_{(m-1) n+1,(m-1) n+1} x_{m}$.

1. Step 1: The first step is Theorem 3.9.

2. Step 2: Deduce that $L\left(A_{1}\right)$ contains a positive column. This involves several steps and the calculations are involved and lengthy even in the $n=3$ case. See the Appendix for a brief explanation.

3. Step 3: Using the expressions $P_{1}, \ldots, P_{m}$, Steps 1 and 2 and assuming that the first column of $L\left(A_{1}\right)$ is positive, we write $L\left(A_{1}\right)$ as

$$
L\left(A_{1}\right)=\left[\begin{array}{ccccc}
P_{1} & \alpha_{1,1} P_{1} & \ldots & \alpha_{1, n-2} P_{1} & \alpha_{1, n-1} P_{1} \\
P_{2} & \alpha_{2,1} P_{2} & \ldots & \alpha_{2, n-2} P_{2} & \alpha_{2, n-1} P_{2} \\
\vdots & \vdots & \ldots & \vdots & \vdots \\
P_{m} & \alpha_{m, 1} P_{m} & \ldots & \alpha_{m, n-2} P_{m} & \alpha_{m, n-1} P_{m}
\end{array}\right] \text {, where } \alpha_{i, j} \in \mathbb{R} .
$$

4. Step 4: Consider the matrix $E:=\left[\begin{array}{cccc}\alpha_{1,1} & \ldots & \alpha_{1, n-2} & \alpha_{1, n-1} \\ \alpha_{2,1} & \ldots & \alpha_{2, n-2} & \alpha_{2, n-1} \\ \vdots & \vdots & \ldots & \vdots \\ \alpha_{m, 1} & \ldots & \alpha_{m, n-2} & \alpha_{m, n-1}\end{array}\right]$. If the matrix $E$ is semipositive, then $L\left(A_{1}\right)$ has rank one. Therefore, $L\left(A_{1}\right)=\left[\begin{array}{c}P_{1} \\ P_{2} \\ \vdots \\ P_{m}\end{array}\right]\left[\begin{array}{llll}1 & \alpha_{1,1} & \ldots & \alpha_{1, n-1}\end{array}\right]$. 
Electronic Journal of Linear Algebra, ISSN 1081-3810

5. Step 5: If the matrix $-E$ is semipositive, whereas $E$ is not, then again $L\left(A_{1}\right)$ has rank one and $L\left(A_{1}\right)=\left[\begin{array}{c}P_{1} \\ P_{2} \\ \vdots \\ P_{m}\end{array}\right]\left[\begin{array}{llll}1 & \alpha_{1,1} & \ldots & \alpha_{1, n-1}\end{array}\right]$

6. Step 6: Verify that $L\left(A_{2}\right)$ has a positive column. If both $E$ and $-E$ are not semipositive, then $L\left(A_{1}\right)$ has rank one. The proof of this goes as follows. Assuming that the second column of $L\left(A_{2}\right)$ is positive, we have $L\left(A_{2}\right)=\left[\begin{array}{cccc}\beta_{1,1} Q_{1} & Q_{1} & \ldots & \beta_{1, n-1} Q_{1} \\ \beta_{2,1} Q_{2} & Q_{2} & \ldots & \beta_{2, n-1} Q_{2} \\ \vdots & \vdots & \ldots & \vdots \\ \beta_{m, 1} Q_{m} & Q_{m} & \ldots & \beta_{m, n-1} Q_{m}\end{array}\right]$. Choose a vector $p \in \mathbb{R}^{m}$ such that $L\left(\left[\begin{array}{rrrr}p_{1} & 0 & \ldots & 0 \\ p_{2} & 0 & \ldots & 0 \\ \vdots & \vdots & \ldots & \vdots \\ p_{m} & 0 & \ldots & 0\end{array}\right]\right)=\left[\begin{array}{ccccc}1 & \alpha_{1,1} & \alpha_{1,2} & \ldots & \alpha_{1, n-1} \\ 1 & \alpha_{2,1} & \alpha_{2,2} & \ldots & \alpha_{2, n-1} \\ \vdots & \vdots & \vdots & \ldots & \vdots \\ 1 & \alpha_{m, 1} & \alpha_{m, 2} & \ldots & \alpha_{m, n-1}\end{array}\right]$. If $A_{2}$ is semipositive, then the ma$\operatorname{trix}\left[\begin{array}{cccc}-p_{1} & 0 & \ldots & 0 \\ -p_{2} & 0 & \ldots & 0 \\ \vdots & \vdots & \ldots & \vdots \\ -p_{m} & 0 & \ldots & 0\end{array}\right]+A_{2}$ is semipositive and so is its image under $L$. Note that it is possible to choose a $y_{2}>0$, so that the first column of the image of the above matrix under $L$ is negative. Let $q \in \mathbb{R}^{m}$ be such that $L\left(\left[\begin{array}{rrrr}p_{1} & 0 & \ldots & 0 \\ p_{2} & 0 & \ldots & 0 \\ \vdots & \vdots & \ldots & \vdots \\ p_{m} & 0 & \ldots & 0\end{array}\right]-y_{2}\left[\begin{array}{ccccc}0 & q_{1} & 0 & \ldots & 0 \\ 0 & q_{2} & 0 & \ldots & 0 \\ \vdots & \vdots & \vdots & \ldots & 0 \\ 0 & q_{m} & \vdots & \ldots & 0\end{array}\right]\right)$ equals $\left[\begin{array}{cccc}1-\beta_{1,1} y_{2} & \alpha_{1,1}-y_{2} & \ldots & \alpha_{1, n-1}-\beta_{1, n-1} y_{2} \\ 1-\beta_{2,1} y_{2} & \alpha_{2,1}-y_{2} & \ldots & \alpha_{2, n-1}-\beta_{2, n-1} y_{2} \\ \vdots & \vdots & \ldots & \vdots \\ 1-\beta_{m, 1} y_{2} & \alpha_{m, 1}-y_{2} & \ldots & \alpha_{m, n-1}-\beta_{m, n-1} y_{2}\end{array}\right]$. It is now possible to choose a sufficiently small $y_{2}>0$ so that the first column of the above matrix is positive and the matrix

$-\left[\begin{array}{ccc}\alpha_{1,1}-y_{2} & \ldots & \alpha_{1, n-1}-\beta_{1, n-1} y_{2} \\ \alpha_{2,1}-y_{2} & \ldots & \alpha_{2, n-1}-\beta_{2, n-1} y_{2} \\ \vdots & \ldots & \vdots \\ \alpha_{m, 1}-y_{2} & \cdots & \alpha_{m, n-1}-\beta_{m, n-1} y_{2}\end{array}\right]$ is semipositive. From the previous case, it follows that

$\alpha_{1,1}-y_{2}=\alpha_{2,1}-y_{2}=\cdots=\alpha_{m, 1}-y_{2}$, which in turn yields $\alpha_{1,1}=\alpha_{2,1}=\cdots=\alpha_{m, 1}=0$. Similarly, we can show that $\alpha_{1, i}=\alpha_{2, i}=\cdots=\alpha_{m, i}=0$ for $i=2, \ldots, n-1$. We finally have $L\left(A_{1}\right)=\left[\begin{array}{c}P_{1} \\ P_{2} \\ \vdots \\ P_{m}\end{array}\right]\left[\begin{array}{llll}1 & 0 & \ldots & 0\end{array}\right]$.

This proves the theorem.

We now prove our main result concerning the structure of an into preserver of semipositivity. 
THEOREM 3.11. For $m \geq n$, let $L$ be an invertible linear map on $M_{m, n}$ such that $L\left(S\left(\mathbb{R}_{+}^{n}, \mathbb{R}_{+}^{m}\right)\right) \subset$ $S\left(\mathbb{R}_{+}^{n}, \mathbb{R}_{+}^{m}\right)$. Then, $L(A)=X A Y$ for all $A \in M_{m, n}$, where $X \in M_{m}$ is an invertible row positive matrix and $Y \in M_{n}$ is an inverse nonnegative matrix.

Proof. We know from Theorem 3.10 that $L\left(A_{i}\right)$ has rank one for each $i=1, \ldots, n$. Suppose the first and

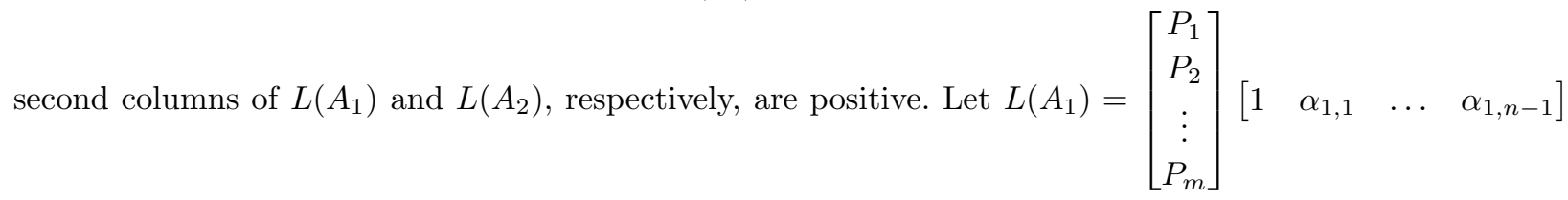
and $L\left(A_{2}\right)=\left[\begin{array}{c}Q_{1} \\ Q_{2} \\ \vdots \\ Q_{m}\end{array}\right]\left[\begin{array}{llll}\alpha_{2,1} & 1 & \ldots & \alpha_{2, n-1}\end{array}\right]$

Claim 1: The vectors $\left(l_{1,1}, l_{1, n+1}, \ldots, l_{1,(m-1) n+1}\right)^{t}$ and $\left(l_{2,2}, l_{2, n+2}, \ldots, l_{2,(m-1) n+2}\right)^{t}$ are linearly dependent. If not, then there will exist two vectors $u=\left(u_{1}, \ldots, u_{m}\right)^{t}$ and $v=\left(v_{1}, \ldots, v_{m}\right)^{t}$ in $\mathbb{R}^{m}$ such that $\left[\begin{array}{llll}l_{2,2} & l_{2, n+2} & \ldots & l_{2,(m-1) n+2} \\ l_{1,1} & l_{1, n+1} & \ldots & l_{1,(m-1) n+1}\end{array}\right]\left[\begin{array}{cc}u_{1} & v_{1} \\ u_{2} & v_{2} \\ \vdots & \vdots \\ u_{m} & v_{m}\end{array}\right]=\left[\begin{array}{cc}1 & 0 \\ 0 & 1\end{array}\right]$. Since $\left[\begin{array}{llll}l_{2,2} & l_{2, n+2} & \ldots & l_{2,(m-1) n+2} \\ l_{1,1} & l_{1, n+1} & \ldots & l_{1,(m-1) n+1}\end{array}\right]$ is a rank two nonnegative matrix, we see that the matrix $B=\left[\begin{array}{ccccc}u_{1} & v_{1} & 0 & \ldots & 0 \\ u_{2} & v_{2} & 0 & \ldots & 0 \\ \vdots & \vdots & \vdots & \ldots & \vdots \\ u_{m} & v_{m} & 0 & \ldots & 0\end{array}\right]$ is an $m \times n$ semipositive matrix. However, $L(B)=\left[\begin{array}{cccc}0 & 0 & \ldots & 0 \\ * & * & \ldots & * \\ \vdots & \vdots & \ldots & * \\ * & * & \ldots & *\end{array}\right]$ is not semipositive. Therefore, there exists a positive real number $\lambda_{1,1}$ such that $\left(l_{2,2}, l_{2, n+2}, \ldots, l_{2,(m-1) n+2}\right)^{t}=\lambda_{1,1}\left(l_{1,1}, l_{1, n+1}, \ldots, l_{1,(m-1) n+1}\right)^{t}$. Proceeding in a similar way, it can be shown that $L\left(A_{2}\right)=\left[\begin{array}{c}\lambda_{1,1} P_{1} \\ \lambda_{2,1} P_{2} \\ \vdots \\ \lambda_{m, 1} P_{m}\end{array}\right]\left[\begin{array}{llll}\alpha_{2,1} & 1 & \ldots & \alpha_{2, n-1}\end{array}\right]$, where $\lambda_{j, 1}>0$ for $j=1, \ldots, m$. More generally, it can be shown that $L\left(A_{i}\right)=\left[\begin{array}{c}\lambda_{1, i-1} P_{1} \\ \lambda_{2, i-1} P_{2} \\ \vdots \\ \lambda_{m, i-1} P_{m}\end{array}\right]\left[\begin{array}{llll}\alpha_{i, 1} & \alpha_{i, 2} & \ldots & \alpha_{i, n}\end{array}\right]$, where some $\alpha_{i, k}>0, i=3,4, \ldots, n$.

Claim 2: $\lambda_{1, i-1}=\lambda_{2, i-1}=\cdots=\lambda_{m, i-1}, i=2, \ldots, n$.

We prove that $\lambda_{1,1}=\lambda_{2,1}$ and skip the remaining arguments, as the idea is the same. Assume that $\lambda_{1,1}>\lambda_{2,1}$. Choose positive numbers $d_{1}, d_{2}$ and form the matrix $B$ defined as 
$\left[\begin{array}{cccc}l_{1,1} & l_{1, n+1} & \ldots & l_{1,(m-1) n+1} \\ l_{n+1,1} & l_{n+1, n+1} & \ldots & l_{n+1,(m-1) n+1} \\ \vdots & \vdots & \ldots & \vdots \\ l_{(m-1) n+1,1} & l_{(m-1) n+1, n+1} & \ldots & l_{(m-1) n+1,(m-1) n+1}\end{array}\right]=\left[\begin{array}{cccccc}d_{1} & \frac{-d_{1}}{\lambda_{1,1}} & 0 & 0 & \ldots & 0 \\ -d_{2} & \frac{d_{2}}{\lambda_{2,1}} & 0 & 0 & \ldots & 0 \\ 0 & 0 & 1 & 0 & \ldots & 0 \\ 0 & 0 & 0 & 1 & \ldots & 0 \\ \vdots & \vdots & \vdots & \vdots & \ddots & \\ 0 & 0 & 0 & 0 & \ldots & 1\end{array}\right]$. It is clear that $B$ is

a semipositive matrix. But $L(B)=\left[\begin{array}{cccc}\left(1-\alpha_{2,1}\right) d_{1} & \left(\alpha_{1,1}-\alpha_{2,1}\right) d_{1} & \ldots & \left(\alpha_{1, n-1}-\alpha_{2, n-1}\right) d_{1} \\ -\left(1-\alpha_{2,1}\right) d_{2} & -\left(\alpha_{1,1}-\alpha_{2,1}\right) d_{2} & \ldots & -\left(\alpha_{1, n-1}-\alpha_{2, n-1}\right) d_{2} \\ * & * & \ldots & * \\ * & * & \ldots & \ldots \\ \vdots & \vdots & \ldots & * \\ * & * & \ldots & *\end{array}\right]$, which

is not semipositive (notice that each $2 \times 2$ submatrix of the matrix formed from the first rows of $L(B)$ is not semipositive). Therefore, $\lambda_{1,1} \leq \lambda_{2,1}$. Similarly, it can be proved that $\lambda_{1,1} \geq \lambda_{2,1}$. Consequently, $\lambda_{1,1}=\lambda_{2,1}$. Proceeding this way, it can be seen that $\lambda_{1, i-1}=\lambda_{2, i-1}=\cdots=\lambda_{m, i-1}$, $i=$ $2, \ldots, n$. We finally have $L(A)=X A Y$ for any $A \in M_{m, n}$, where $X$ and $Y$ are the matrices $X=\left[\begin{array}{cccc}l_{1,1} & l_{1, n+1} & \cdots & l_{1,(m-1) n+1} \\ l_{n+1,1} & l_{n+1, n+1} & \cdots & l_{n+1,(m-1) n+1} \\ \vdots & \vdots & \ldots & \vdots \\ l_{(m-1) n+1,1} & l_{(m-1) n+1, n+1} & \cdots & l_{(m-1) n+1,(m-1) n+1}\end{array}\right], \quad$ a row positive matrix and $Y=\left[\begin{array}{cccc}1 & \alpha_{1,1} & \ldots & \alpha_{1, n-1} \\ \lambda_{1,1} \alpha_{2,1} & \lambda_{1,1} & \ldots & \lambda_{1,1} \alpha_{2, n-1} \\ \lambda_{1,2} \alpha_{3,1} & \lambda_{1,2} \alpha_{3,2} & \ldots & \lambda_{1,2} \alpha_{3, n} \\ \vdots & \vdots & \ldots & \vdots \\ \lambda_{1, n-1} \alpha_{n, 1} & \lambda_{1, n-1} \alpha_{n, 2} & \ldots & \lambda_{1, n-1} \alpha_{n, n}\end{array}\right]$. Since $L$ preserves semipositivity, it follows from Theorem 2.4 of [9] that $Y$ is inverse nonnegative.

Summarizing everything, we have proved the following theorem.

THEOREM 3.12. Let $L$ be an invertible linear map on $M_{m, n}$ such that $L\left(S\left(\mathbb{R}_{+}^{n}, \mathbb{R}_{+}^{m}\right)\right) \subset S\left(\mathbb{R}_{+}^{n}, \mathbb{R}_{+}^{m}\right)$ ( $m \geq n \geq 2$ ). Then, $L(A)=X A Y$ for all $A \in M_{m, n}$, where $X \in M_{m}$ is an invertible row positive matrix and $Y \in M_{n}$ is an inverse nonnegative matrix if and only if each $A_{i}$ that is semipositive gets mapped to a rank one matrix.

3.2. The proper cones case. We begin this section with useful results needed in subsequent sections. These include the preserver properties of $S\left(K_{1}, K_{2}\right)$ and $M S\left(K_{1}, K_{2}\right)$ under a specific map and the existence of a basis for $M_{m, n}$ from either of the above sets. Recall that all the cones are assumed to be proper.

3.2.1. Useful results. We begin with the following result known as a Theorem of the Alternative.

Theorem 3.13 ([5, Theorem 2.8]). For proper cones $K_{1}$ and $K_{2}$ in $\mathbb{R}^{n}$ and $\mathbb{R}^{m}$, respectively, and an $m \times n$ matrix $A$, one and only one of the following alternatives holds.

(a) There exists $x \in K_{1}$ such that $A x \in K_{2}^{\circ}$.

(b) There exists $0 \neq y \in K_{2}^{*}$ such that $-A^{t} y \in K_{1}^{*}$. 
Electronic Journal of Linear Algebra, ISSN 1081-3810

It is a fairly well-known result that the closure of the interior of a convex subset $K$ of $\mathbb{R}^{n}$ equals the closure of $K$. We shall use this in the proofs later on. For completeness, we present a proof.

Lemma 3.14. Let $F$ be a convex set in $\mathbb{R}^{n}$ with a nonempty interior. Then, $\overline{F^{\circ}}=\bar{F}$.

Proof. We only need to prove that $\bar{F} \subset \overline{F^{\circ}}$. If $a \in F, b \in \bar{F}$, then the set $\{(1 / n) a+(1-1 / n) b: n \in \mathbb{N}\}$ is contained in the interior of $F$. Moreover, elements of the above set converge to $b$. Thus, $b \in \overline{F^{\circ}}$.

The following is a well-known fact concerning nonnegative matrices.

Lemma 3.15 ([10, Corollary 3.3]). Let $K_{1}$ and $K_{2}$ be proper cones in $\mathbb{R}^{n}$ and $\mathbb{R}^{m}$, respectively, and $S: \mathbb{R}^{n} \rightarrow \mathbb{R}^{m}$ be a linear map such that $S\left(K_{1}\right) \subseteq K_{2}$. Then $S^{t}\left(K_{2}^{*}\right) \subseteq K_{1}^{*}$.

We now prove that given any element $v$ of a proper cone $K$, there is a subcone $K_{1}$ of $K$ which is simplicial and containing the point $v$.

Lemma 3.16. Let $K$ be a proper cone in $\mathbb{R}^{n}$ and $v \in K$. Then there exists an invertible $T \in \pi\left(\mathbb{R}_{+}^{n}, K\right)$ such that $T x=v$ for some $x \in \mathbb{R}_{+}^{n}$.

Proof. If $n=2$, then $K$ is a simplicial cone. Therefore, $T\left(\mathbb{R}_{+}^{2}\right)=K$ for some invertible $T \in M_{2}(\mathbb{R})$. In such a case, the result is obvious.

Let $n \geq 3$ and $v \in K$. Since $K$ is a proper cone, there exits $v_{2} \in K$ which is linearly independent of $v$. Suppose for every $z \in K, z=\alpha v_{2}+\beta v$ for some $\alpha, \beta \in \mathbb{R}$. Then $K \subsetneq \operatorname{span}\left\{v, v_{2}\right\}$, a proper subspace of $\mathbb{R}^{n}$. Since such a subspace has empty interior and $K^{\circ} \neq \emptyset$, we get a contradiction. Thus, there exists $v_{3} \in K$ such that $\left\{v, v_{2}, v_{3}\right\}$ is linearly independent. Proceeding by induction, we get a basis $\left\{v, v_{2}, \ldots, v_{n}\right\}$ for $\mathbb{R}^{n}$ such that $v, v_{2}, \ldots, v_{n} \in K$. Take $T=\left[\begin{array}{lll}v & v_{2} \cdots v_{n}\end{array}\right]$. Then, $T x=v$, where $x=\left[\begin{array}{lll}1 & 0 & \cdots\end{array}\right]^{t} \in \mathbb{R}_{+}^{n}$. Since $K$ is a convex cone, $T \in \pi\left(\mathbb{R}_{+}^{n}, K\right)$. This completes the proof.

Let us recall the following result from [1].

Theorem 3.17 ([1, Theorem 2.4]). For proper cones $K_{1}, K_{2}$ in $\mathbb{R}^{n}$, let $S \in \pi\left(K_{1}, K_{2}\right)$ be an invertible linear map on $\mathbb{R}^{n}$. If a matrix $A$ is $K_{1}$-semipositive, then the matrix $B=S A S^{-1}$ is $K_{2}$-semipositive. Conversely, if the cones are self-dual and if $C$ is $K_{2}$-semipositive, then there exists a $K_{1}$-semipositive matrix A such that $C=\left(S^{t}\right)^{-1} A S^{t}$.

The following will be used subsequently. We state it without proof.

Lemma 3.18. Let $K_{1}$ and $K_{2}$ be proper cones in $\mathbb{R}^{n}$ and $\mathbb{R}^{m}$, respectively. Then the following hold:

1. Let $Q_{1} \in \pi\left(K_{2}, \mathbb{R}_{+}^{m}\right)$ with $Q_{1}\left(\left(K_{2}\right)^{\circ}\right) \subseteq\left(\mathbb{R}_{+}^{m}\right)^{\circ}$ and an invertible $Q_{2} \in \pi\left(K_{1}, \mathbb{R}_{+}^{n}\right)$. If $A \in S\left(K_{1}, K_{2}\right)$, then $Q_{1} A Q_{2}^{-1} \in S\left(\mathbb{R}_{+}^{n}, \mathbb{R}_{+}^{m}\right)$.

2. Let $S_{1} \in \pi\left(\mathbb{R}_{+}^{m}, K_{2}\right)$ with $S_{1}\left(\left(\mathbb{R}_{+}^{m}\right)^{\circ}\right) \subseteq\left(K_{2}\right)^{\circ}$ and an invertible $S_{2} \in \pi\left(\mathbb{R}_{+}^{n}, K_{1}\right)$. If $B \in S\left(\mathbb{R}_{+}^{n}, \mathbb{R}_{+}^{m}\right)$, then $S_{1} B S_{2}^{-1} \in S\left(K_{1}, K_{2}\right)$.

The following two results are similar to that of Theorem 3.17 for $M S\left(K_{1}, K_{2}\right)$.

Lemma 3.19. For $m>n$, let $K_{1}$ and $K_{2}$ be proper cones in $\mathbb{R}^{n}$ and $\mathbb{R}^{m}$, respectively, with $K_{2}$ simplicial.

1. If $S$ and $T$ are invertible maps on $\mathbb{R}^{n}$ and $\mathbb{R}^{m}$ such that $S \in \pi\left(\mathbb{R}_{+}^{n}, K_{1}\right)$ and $T\left(\mathbb{R}_{+}^{m}\right)=K_{2}$, then $T A S^{-1} \in M S\left(K_{1}, K_{2}\right)$ whenever $A \in M_{m, n}$ is minimally semipositive.

2. If $S$ and $T$ are invertible maps on $\mathbb{R}^{n}$ and $\mathbb{R}^{m}$ such that $S \in \pi\left(\mathbb{R}_{+}^{n}, K_{1}^{*}\right)$ and $T\left(\mathbb{R}_{+}^{m}\right)=K_{2}$, then $T^{-1} B\left(S^{t}\right)^{-1}$ is minimally semipositive whenever $B \in M S\left(K_{1}, K_{2}\right)$. 
Proof. We prove only the first statement as the proof of the second statement is similar. Observe that $T A \in S\left(\mathbb{R}_{+}^{n}, K_{2}\right)$. Let $B \in M_{n, m}$ be a nonnegative left inverse of $A$. Then $S B T^{-1} \in \pi\left(K_{2}, K_{1}\right)$ is a left inverse of $T A S^{-1}$. Therefore, it is enough to prove that $T A S^{-1} \in S\left(K_{1}, K_{2}\right)$. Suppose $T A S^{-1} \notin S\left(K_{1}, K_{2}\right)$. By the Theorem of the Alternative (Theorem 3.13), there exists $0 \neq x \in K_{2}^{*}$ such that $-\left(S^{t}\right)^{-1} A^{t} T^{t} x \in K_{1}^{*}$. We then have $-A^{t} T^{t} x \in \mathbb{R}_{+}^{n}$ which implies that $T A \notin S\left(\mathbb{R}_{+}^{n}, K_{2}\right)$, a contradiction.

Observe that we need not assume that the cone $K_{2}$ is simplicial if $m=n$, where we have the following result: For proper cones $K_{1}$ and $K_{2}$ in $\mathbb{R}^{n}$, let $S \in \pi\left(\mathbb{R}_{+}^{n}, K_{1}\right)$ and $T \in \pi\left(\mathbb{R}_{+}^{n}, K_{2}^{*}\right)$ be invertible matrices. Then, $\left(T^{t}\right)^{-1} A S^{-1} \in M S\left(K_{1}, K_{2}\right)$ whenever $A$ is minimally semipositive. Similarly, if $S \in \pi\left(\mathbb{R}_{+}^{n}, K_{1}^{*}\right)$ and $T \in \pi\left(\mathbb{R}_{+}^{n}, K_{2}\right)$ are invertible matrices, then $T^{-1} B\left(S^{t}\right)^{-1}$ is minimally semipositive whenever $B \in$ $M S\left(K_{1}, K_{2}\right)$. We skip the proof as it is similar to the above lemma.

Before proceeding further, let us mention the following useful results that follow from Lemmas 3.18 and 3.19 .

Observation 3.20. For $A \in M_{m, n}$ and proper cones $K_{1}$ and $K_{2}$ in $\mathbb{R}^{n}$ and $\mathbb{R}^{m}$, respectively, the following hold:

1. There exists $B, C \in S\left(K_{1}, K_{2}\right)$ such that $A=B+C$.

2. There exists $C_{1}, C_{2} \in M S\left(K_{1}, K_{2}\right)$ such that $A=C_{1}-C_{2}$, if in addition the cone $K_{2}$ is simplicial when $m>n$.

We end this subsection by proving that $M_{m, n}$ contains a basis from $S\left(K_{1}, K_{2}\right)$ and $M S\left(K_{1}, K_{2}\right)$. The following result was proved recently by P. N. Choudhury et al. [6].

TheOREM 3.21 ([6, Theorem 3.1]). There is a basis of minimally semipositive matrices for $M_{m, n}$, $m \geq n$.

Below is the proof that $M_{m, n}$ contains a basis from both $S\left(K_{1}, K_{2}\right)$ and $M S\left(K_{1}, K_{2}\right)$.

THEOREM 3.22. Given proper cones $K_{1}$ and $K_{2}$ in $\mathbb{R}^{n}$ and $\mathbb{R}^{m}$, respectively, the following hold:

1. $S\left(K_{1}, K_{2}\right)$ contains a basis for $M_{m, n}$.

2. $M S\left(K_{1}, K_{2}\right)$ contains a basis for $M_{m, n}$, if in addition the cone $K_{2}$ is simplicial, when $m>n$.

Proof. (1) From [9], we know that $S\left(\mathbb{R}_{+}^{n}, \mathbb{R}_{+}^{m}\right)$ contains a basis for $M_{m, n}$. Let $\left\{A_{i j}\right\} \subset S\left(\mathbb{R}_{+}^{n}, \mathbb{R}_{+}^{m}\right)$ be a basis for $M_{m, n}$. Then by Lemma 3.18(2), $\left\{B_{i j}=T A_{i j} S^{-1}\right\} \subset S\left(K_{1}, K_{2}\right)$ will be a basis for $M_{m, n}$, where $S \in \pi\left(\mathbb{R}_{+}^{n}, K_{1}\right)$ and $T \in \pi\left(\mathbb{R}_{+}^{m}, K_{2}\right)$ are invertible matrices with $T\left(\left(\mathbb{R}_{+}^{m}\right)^{\circ}\right) \subseteq\left(K_{2}\right)^{\circ}$.

(2) By Theorem 3.21, let $\left\{\widetilde{A_{i j}}\right\}$ be a collection of minimally semipositive matrices that form a basis for $M_{m, n}$. Then by Lemma 3.19, $\left\{\widetilde{B_{i j}}=T \widetilde{A_{i j}} S^{-1}\right\} \subset M S\left(K_{1}, K_{2}\right)$ is a basis for $M_{m, n}$, where $S \in \pi\left(\mathbb{R}_{+}^{n}, K_{1}\right)$ and $T\left(\mathbb{R}_{+}^{m}\right)=K_{2}$ are invertible matrices.

We are now in a position to tackle preservers of $S\left(K_{1}, K_{2}\right)$. Recall the following.

Definition 3.23. A linear map $L$ on $M_{m, n}$ is an onto preserver of $\mathcal{S}$ if $L(\mathcal{S})=\mathcal{S}$.

We shall use the following lemma in our proofs (see [8] for details).

LEMMA 3.24. If $\mathcal{S}$ contains a basis for $M_{m, n}$, then $L$ is an onto preserver of $\mathcal{S}$ if and only if $L$ and $L^{-1}$ are into preservers of $\mathcal{S}$. 
3.2.2. Preservers of $S\left(K_{1}, K_{2}\right)$. We begin this section with results that will be used in the proof of Theorem 3.29.

Lemma 3.25. Let $X \in M_{n}$. If $S X T \in \pi\left(\mathbb{R}_{+}^{n}\right)$ for all invertible matrices $T \in \pi\left(\mathbb{R}_{+}^{n}, K\right)$ and $S \in \pi\left(K, \mathbb{R}_{+}^{n}\right)$, then $X \in \pi(K)$.

Proof. Let $v \in K$. By Lemma 3.16, there exists $T \in \pi\left(\mathbb{R}_{+}^{n}, K\right)$ such that $T x=v$ for some $x \in \mathbb{R}_{+}^{n}$. We have $S X T x=S X v \in \mathbb{R}_{+}^{n}$ for all invertible $S \in \pi\left(K, \mathbb{R}_{+}^{n}\right)$. Therefore, $\langle S X v, u\rangle=\left\langle X v, S^{t} u\right\rangle \geq 0$ for all $u \in \mathbb{R}_{+}^{n}$ and all invertible $S \in \pi\left(K, \mathbb{R}_{+}^{n}\right)$. Let us take $p \in K^{*}$. By Lemma 3.16, there exists an invertible $T_{1} \in \pi\left(\mathbb{R}_{+}^{n}, K^{*}\right)$ such that $T_{1} y=p$ for some $y \in \mathbb{R}_{+}^{n}$. In particular, $\left\langle X v, T_{1} y\right\rangle=\langle X v, p\rangle \geq 0$. Therefore, we get $X v \in K$.

Recall that a square matrix $A$ is said to be row positive if $A$ is nonnegative with a nonzero entry in each row.

Lemma 3.26. Let $X \in M_{n}$. If $S X T$ is row positive for all $T \in \pi\left(\mathbb{R}_{+}^{n}, K\right)$ and $S \in \pi\left(K, \mathbb{R}_{+}^{n}\right)$ with $T\left(\left(\mathbb{R}_{+}^{n}\right)^{\circ}\right) \subseteq K^{\circ}$ and $S\left(K^{\circ}\right) \subseteq\left(\mathbb{R}_{+}^{n}\right)^{\circ}$, then $X\left(K^{\circ}\right) \subseteq K^{\circ}$.

Proof. Let $v \in K^{\circ}$. Take $T=\left[\frac{1}{n} v \cdots \frac{1}{n} v\right]$. We see that $T \in \pi\left(\mathbb{R}_{+}^{n}, K\right)$ with $T\left(\left(\mathbb{R}_{+}^{n}\right)^{\circ}\right) \subseteq K^{\circ}$ and $T x=v$, where $x=[1 \cdots 1]^{t} \in\left(\mathbb{R}_{+}^{n}\right)^{\circ}$. We have $S X T x=S X v \in\left(\mathbb{R}_{+}^{n}\right)^{\circ}$ for all $S \in \pi\left(K, \mathbb{R}_{+}^{n}\right)$ with $S\left(K^{\circ}\right) \subseteq\left(\mathbb{R}_{+}^{n}\right)^{\circ}$. Then, $\langle S X v, u\rangle=\left\langle X v, S^{t} u\right\rangle>0$ for all $0 \neq u \in \mathbb{R}_{+}^{n}$ and $S \in \pi\left(K, \mathbb{R}_{+}^{n}\right)$ with $S\left(K^{\circ}\right) \subseteq\left(\mathbb{R}_{+}^{n}\right)^{\circ}$. Let us take $0 \neq p \in K^{*}$ and $T_{1}=\left[\begin{array}{ll}p & q\end{array} \cdots q\right]$, where $q \in\left(K^{*}\right)^{\circ}$. It is easy to verify that $T_{1} \in \pi\left(\mathbb{R}_{+}^{n}, K^{*}\right)$ with $T\left(\left(\mathbb{R}_{+}^{n}\right)^{\circ}\right) \subseteq K^{\circ}$ and $T_{1} y=p$, where $y=\left[\begin{array}{llll}1 & 0 & \cdots & 0\end{array}\right]^{t} \in \mathbb{R}_{+}^{n}$. In particular, $\left\langle X v, T_{1} y\right\rangle=\langle X v, p\rangle>0$. Therefore, we get $X v \in K^{\circ}$.

The main theorems of this section are proved below.

TheOREM 3.27. Let $S_{2} \in \pi\left(\mathbb{R}_{+}^{n}, K_{1}\right)$ and $Q_{2} \in \pi\left(K_{1}, \mathbb{R}_{+}^{n}\right)$ be invertible matrices and $S_{1} \in \pi\left(\mathbb{R}_{+}^{m}, K_{2}\right)$ and $Q_{1} \in \pi\left(K_{2}, \mathbb{R}_{+}^{m}\right)$ with $S_{1}\left(\left(\mathbb{R}_{+}^{m}\right)^{\circ}\right) \subseteq K_{2}^{\circ}$ and $Q_{1}\left(K_{2}^{\circ}\right) \subseteq\left(\mathbb{R}_{+}^{m}\right)^{\circ}$, respectively. Let $T_{1}(A)=Q_{1} A Q_{2}^{-1}$ and $T_{2}(A)=S_{1} A S_{2}^{-1}$. If $L: M_{m, n} \rightarrow M_{m, n}$ is an into preserver of $S\left(K_{1}, K_{2}\right)$, then $L_{1}=T_{1} L T_{2}$ is an into preserver of $S\left(\mathbb{R}_{+}^{n}, \mathbb{R}_{+}^{m}\right)$.

Proof. Let $A \in S\left(\mathbb{R}_{+}^{n}, \mathbb{R}_{+}^{m}\right)$. By Lemma 3.18, $T_{2}(A) \in S\left(K_{1}, K_{2}\right)$. Then $L T_{2}(A) \in S\left(K_{1}, K_{2}\right)$, since $L\left(S\left(K_{1}, K_{2}\right)\right) \subset S\left(K_{1}, K_{2}\right)$. By Lemma 3.18, we finally have $T_{1} L T_{2}(A) \in S\left(\mathbb{R}_{+}^{n}, \mathbb{R}_{+}^{m}\right)$.

REMARK 3.28. Suppose the map $L$ as well as the matrices $S_{1}$ and $Q_{1}$ are invertible (so that the maps $T_{1}$ and $T_{2}$ are invertible), then the map $L_{1}$ is an invertible linear preserver of semipositivity. It then follows from Theorem 3.11 that $L_{1}(A)=X A Y$ for every $A \in M_{m, n}$ for some invertible row positive matrix $X$ and an inverse nonnegative matrix $Y$. This also yields that $L(A)=\widetilde{X} A \widetilde{Y}$ for every $A \in M_{m, n}$ for some matrices $\widetilde{X}$ and $\widetilde{Y}$ of appropriate sizes. This gives us a motivation to study preserver properties of the map $A \mapsto X A Y$ for appropriate $X$ and $Y$. We however wish to emphasize that no invertibility assumption is made in the following result.

Theorem 3.29. Let $L(A)=X A Y$ be a linear map on $M_{m, n}$, where $X \in M_{m}$ and $Y \in M_{n}$ are fixed. $L$ is an into preserver of $S\left(K_{1}, K_{2}\right)$ if and only if either $X\left(K_{2}^{\circ}\right) \subseteq K_{2}^{\circ}$ and $Y$ is $K_{1}$-inverse nonnegative or $-X\left(K_{2}^{\circ}\right) \subseteq K_{2}^{\circ}$ and $-Y$ is $K_{1}$-inverse nonnegative.

Proof. Suppose $X\left(K_{2}^{\circ}\right) \subseteq K_{2}^{\circ}$ and $Y$ is $K_{1}$-inverse nonnegative, then $X A Y \in S\left(K_{1}, K_{2}\right)$ whenever $A \in S\left(K_{1}, K_{2}\right)$. If $-X\left(K_{2}^{\circ}\right) \subseteq K_{2}^{\circ}$ and $-Y$ is $K_{1}$-inverse nonnegative, then $L(A)=(-X) A(-Y)=X A Y \in$ $S\left(K_{1}, K_{2}\right)$ whenever $A \in S\left(K_{1}, K_{2}\right)$. 
Conversely, by Theorem 3.27, the map $L_{1}(A)=T_{1} L T_{2}(A)=Q_{1} X S_{1} A S_{2}^{-1} Y Q_{2}^{-1}$ is an into preserver of $S\left(\mathbb{R}_{+}^{n}, \mathbb{R}_{+}^{m}\right.$ ), for all $Q_{1}, Q_{2}, S_{1}, S_{2}$ (all of them satisfying the assumptions of Theorem 3.27). By Theorem 2.4 of [9], either $Q_{1} X S_{1}$ is row positive and $S_{2}^{-1} Y Q_{2}^{-1}$ is inverse nonnegative or $-Q_{1} X S_{1}$ is row positive and $-S_{2}^{-1} Y Q_{2}^{-1}$ is inverse nonnegative. By using Lemmas 3.25 and 3.26, we finally have either $X\left(K_{2}^{\circ}\right) \subseteq K_{2}^{\circ}$ and $Y$ is $K_{1}$-inverse nonnegative or $-X\left(K_{2}^{\circ}\right) \subseteq K_{2}^{\circ}$ and $-Y$ is $K_{1}$-inverse nonnegative.

The following corollary follows from Theorems 3.22, 3.29, and Lemma 3.24.

Corollary 3.30. The linear map $L(A)=X A Y$ is an onto preserver of $S\left(K_{1}, K_{2}\right)$ if and only if $X\left(K_{2}\right)=K_{2}$ and $Y\left(K_{1}\right)=K_{1}$, or $-X\left(K_{2}\right)=K_{2}$ and $-Y\left(K_{1}\right)=K_{1}$.

Proof. We know that there is a basis for $M_{m, n}$ from $S\left(K_{1}, K_{2}\right)$. Since $L$ is an onto preserver of $S\left(K_{1}, K_{2}\right)$, both $L$ and its inverse are into preservers of $S\left(K_{1}, K_{2}\right)$. Therefore, by the previous theorem, $X\left(K_{2}^{\circ}\right) \subseteq K_{2}^{\circ}$ and $Y\left(K_{1}^{\circ}\right) \subseteq K_{1}^{\circ}\left(\right.$ or $-X\left(K_{2}^{\circ}\right) \subseteq K_{2}^{\circ}$ and $\left.-Y\left(K_{1}^{\circ}\right) \subseteq K_{1}^{\circ}\right)$. Moreover, $X$ and $Y$ (or $-X$ and $-Y$ ) are $K_{2}$-inverse nonnegative and $K_{1}$-inverse nonnegative, respectively. Since $K^{\circ}$ is dense in $K$ by Lemma 3.14, this shows one implication.

Conversely, if $X\left(K_{2}\right)=K_{2}$ and $Y\left(K_{1}\right)=K_{1}$, then $L(A)=X A Y$ is an into preserver of $S\left(K_{1}, K_{2}\right)$ and so is $L^{-1}(A)=X^{-1} A Y^{-1}$. Thus, $L$ is an onto preserver of $S\left(K_{1}, K_{2}\right)$.

3.2.3. Preservers of $M S\left(K_{1}, K_{2}\right)$. We now turn our attention to linear maps $L$ that preserve the set $M S\left(K_{1}, K_{2}\right)$. We start with the following result on nonnegativity.

Lemma 3.31. Let $X \in M_{n}$. If $S^{-1} X\left(T^{t}\right)^{-1} \in \pi\left(\mathbb{R}_{+}^{n}\right)$, for all invertible $S \in \pi\left(\mathbb{R}_{+}^{n}, K\right)$ and $T \in$ $\pi\left(\mathbb{R}_{+}^{n}, K^{*}\right)$, then $X \in \pi(K)$.

Proof. Let $x \in K$. As $K \subset\left(T^{t}\right)^{-1}\left(\mathbb{R}_{+}^{n}\right),\left(T^{t}\right)^{-1} x=v$ for some $x \in \mathbb{R}_{+}^{n}$. We get $\left\langle S^{-1} X v, u\right\rangle=$ $\left\langle X v,\left(S^{t}\right)^{-1} u\right\rangle \geq 0$ for all $u \in \mathbb{R}_{+}^{n}$. Since $K^{*} \subset\left(S^{t}\right)^{-1}\left(\mathbb{R}_{+}^{n}\right)$, it follows that $X v \in K$.

Our main result is the following.

Theorem 3.32. Let $S_{1} \in \pi\left(\mathbb{R}_{+}^{n}, K_{1}^{*}\right), S_{2} \in \pi\left(\mathbb{R}_{+}^{m}, K_{2}\right), Q_{1} \in \pi\left(\mathbb{R}_{+}^{n}, K_{1}\right)$ and $Q_{2} \in \pi\left(\mathbb{R}_{+}^{m}, K_{2}\right)$ be invertible matrices. Assume further that $Q_{2}\left(R_{+}^{m}\right)=K_{2}$ and $S_{2}\left(R_{+}^{m}\right)=K_{2}$. Let $P_{1}(A)=S_{2}^{-1} A\left(S_{1}^{t}\right)^{-1}$ and $P_{2}(A)=Q_{2} A Q_{1}^{-1}$. If $L: M_{m, n} \rightarrow M_{m, n}$ is an into preserver of $M S\left(K_{1}, K_{2}\right)$, then $L_{2}=P_{1} L P_{2}$ is an into preserver of minimally semipositive matrices.

Proof. Notice that $L$ and consequently $L_{2}$ are invertible maps. Let $A$ be minimally semipositive. By Lemma 3.19, $P_{2}(A) \in M S\left(K_{1}, K_{2}\right)$. Then $L P_{2}(A) \in M S\left(K_{1}, K_{2}\right)$, since $L\left(M S\left(K_{1}, K_{2}\right)\right) \subset M S\left(K_{1}, K_{2}\right)$. Again, by Lemma 3.19, we have $P_{1} L P_{2}(A)$ is minimally semipositive.

Similar to the previous section, we now focus our attention to the map $L(A)=X A Y$. We have a complete answer in this case too. We discuss the cases $n<m$ and $n=m$ separately.

TheOREM 3.33. Let $L(A)=X A Y$ be a linear map on $M_{m, n}$ with $n<m$ (where $\left.n \geq 2\right)$ for fixed $X \in M_{m}$ and $Y \in M_{n}$. Then $L$ is an into preserver of $M S\left(K_{1}, K_{2}\right)$ if and only if $X\left(K_{2}\right)=K_{2}$ and $Y$ is $K_{1}$-inverse nonnegative, or $-X\left(K_{2}\right)=K_{2}$ and $-Y$ is $K_{1}$-inverse nonnegative.

Proof. If $A \in M S\left(K_{1}, K_{2}\right)$, then $A$ has a $\left(K_{2}, K_{1}\right)$-nonnegative left inverse. Let $B$ be a $\left(K_{2}, K_{1}\right)$ nonnegative left inverse for $A$. If $X\left(K_{2}\right)=K_{2}$ and $Y$ is $K_{1}$-inverse nonnegative, $Y^{-1} B X^{-1}$ is $\left(K_{2}, K_{1}\right)$ nonnegative and a left inverse for $X A Y$. Since $X A Y \in S\left(K_{1}, K_{2}\right)$, it follows that $X A Y \in M S\left(K_{1}, K_{2}\right)$. 
Conversely, suppose that $L(A)$ is an into preserver of $M S\left(K_{1}, K_{2}\right)$. By Theorem 3.32, $L_{2}(A)=P_{1} L P_{2}(A)$ $=\left(S_{2}\right)^{-1} X Q_{2} A Q_{1}^{-1} Y\left(S_{1}^{t}\right)^{-1}$ is minimally semipositive whenever $A$ is minimally semipositive, where $S_{1}, S_{2}$, $Q_{1}$, and $Q_{2}$ satisfy the assumptions of Theorem 3.32. By Theorem 2.11 of [9], either $\left(S_{2}\right)^{-1} X Q_{2}$ is monomial and $Q_{1}^{-1} Y\left(S_{1}^{t}\right)^{-1}$ is inverse nonnegative or $-\left(S_{2}\right)^{-1} X Q_{2}$ is monomial and $-Q_{1}^{-1} Y\left(S_{1}^{t}\right)^{-1}$ is inverse nonnegative. The result now follows from Lemmas 3.25 and 3.31.

As in the case of onto preservers of $M S\left(K_{1}, K_{2}\right)$, we have the following corollary. We assume again that $n<m$ and that the cone $K_{2}$ is simplicial.

Corollary 3.34. The linear map $L(A)=X A Y$ is an onto preserver of $M S\left(K_{1}, K_{2}\right)$ if and only if $X\left(K_{2}\right)=K_{2}$ and $Y\left(K_{1}\right)=K_{1}$, or $-X\left(K_{2}\right)=K_{2}$ and $-Y\left(K_{1}\right)=K_{1}$.

Proof. Suppose the map $L$ is an onto preserver of $M S\left(K_{1}, K_{2}\right)$. Then $\mathrm{L}$ must be invertible and both $L$ and $L^{-1}$ are into preservers of $M S\left(K_{1}, K_{2}\right)$. By the previous theorem, one implication follows.

Conversely, if $X\left(K_{2}\right)=K_{2}$ and $Y\left(K_{1}\right)=K_{1}$, then obviously $L$ is an onto preserver of $M S\left(K_{1}, K_{2}\right)$, since for every $B \in M S\left(K_{1}, K_{2}\right)$, we can set $A=X^{-1} B Y^{-1} \in M S\left(K_{1}, K_{2}\right)$, so that $L(A)=B$ (see also Theorem 2.11 of [9]).

The $n=m$ case is presented below, the into and onto separately. The proof is omitted as it is similar to that of Theorem 3.33 and follows from Theorem 3.32, Lemmas 3.25 and 3.31, and Theorem 2.10 of [7]. Note that we need not assume simpliciality of the cone $K_{2}$ in this case.

Theorem 3.35. Let $L(A)=X A Y$ be a linear map on $M_{n}$ for fixed $X, Y \in M_{n}$. Then $L$ is an into preserver of $M S\left(K_{1}, K_{2}\right)$ if and only if $X$ is $K_{2}$-inverse nonnegative and $Y$ is $K_{1}$-inverse nonnegative or $-X$ is $K_{2}$-inverse nonnegative and $-Y$ is $K_{1}$-inverse nonnegative.

Corollary 3.36. Let $L(A)=X A Y$ be a linear map on $M_{n}$ for fixed $X, Y \in M_{n}$. The map $L$ is an onto preserver of $M S\left(K_{1}, K_{2}\right)$ if and only if $X\left(K_{2}\right)=K_{2}$ and $Y\left(K_{1}\right)=K_{1}$, or $-X\left(K_{2}\right)=K_{2}$ and $-Y\left(K_{1}\right)=K_{1}$.

3.2.4. General onto preservers of $S\left(K_{1}, K_{2}\right)$. We now turn our attention to general onto preservers of $S\left(K_{1}, K_{2}\right)$. Our main result is the following.

THeOREM 3.37. Let $L$ be a linear map on $M_{m, n}$. If $L$ is an onto preserver of $S\left(K_{1}, K_{2}\right)$, then $L(A)=$ $\widetilde{X} A \widetilde{Y}$ for all $A \in M_{m, n}$, where $\widetilde{X}\left(K_{2}\right)=K_{2}$ and $\widetilde{Y}\left(K_{1}\right)=K_{1}$.

Proof. By Theorem 3.27, we know that for invertible maps $T_{1}$ and $T_{2}$, the map $L_{1}=T_{1} L T_{2}$ is an invertible into linear preserver of $S\left(\mathbb{R}_{+}^{n}, \mathbb{R}_{+}^{m}\right)$. From Theorem 3.11, we infer that $L_{1}(A)=X A Y$ for an invertible row positive matrix $X$ and an inverse nonnegative matrix $Y$. It follows that $L(A)=\widetilde{X} A \widetilde{Y}$ for some $\widetilde{X} \in M_{m}$ and $\widetilde{Y} \in M_{n}$. Finally, Corollary 3.30 yields the desired conclusion.

The following result was proved by A. Chandrashekaran et al.

Theorem 3.38 ([2, Theorem 2.3]). Let $A \in M_{m, n}$ and let $K_{1}, K_{2}$ be proper cones in $\mathbb{R}^{n}$ and $\mathbb{R}^{m}$, respectively. If $A+B \in S\left(K_{1}, K_{2}\right)$ for every $B \in S\left(K_{1}, K_{2}\right)$, then $A \in \pi\left(K_{1}, K_{2}\right)$.

We present below connections between onto preservers of $S\left(K_{1}, K_{2}\right)$ and other preserver properties of maps related to $L$. We begin with the following result. 
LEMMA 3.39. Suppose that $L$ is an onto linear preserver of $S\left(K_{1}, K_{2}\right)$. Then $L$ is an automorphism of the cone $\pi\left(K_{1}, K_{2}\right)$.

Proof. The proof can be found in Theorem 2.6 of [2], by making suitable modifications.

We now prove that if $L$ is an onto preserver of $S\left(K_{1}, K_{2}\right)$, then a map that is equivalent to $L$ will be a preserver of $\pi\left(\mathbb{R}_{+}^{n}, \mathbb{R}_{+}^{m}\right)$.

ThEOREM 3.40. Let $S_{1} \in \pi\left(\mathbb{R}_{+}^{n}, K_{1}\right), S_{2} \in \pi\left(\mathbb{R}_{+}^{n}, K_{1}^{*}\right), T_{1} \in \pi\left(\mathbb{R}_{+}^{m}, K_{2}^{*}\right)$, and $T_{2} \in \pi\left(\mathbb{R}_{+}^{m}, K_{2}\right)$ be invertible matrices. Let $\widetilde{T_{1}}(A)=T_{1}^{t} A S_{1}$ and $\widetilde{T_{2}}(A)=T_{2} A S_{2}^{t}$. If $L: M_{m, n} \rightarrow M_{m, n}$ is an onto preserver of $S\left(K_{1}, K_{2}\right)$, then $\widetilde{L_{1}}=\widetilde{T_{1}} L \widetilde{T}_{2}$ is an into preserver of $\pi\left(\mathbb{R}_{+}^{n}, \mathbb{R}_{+}^{m}\right)$.

Proof. Let $A \in \pi\left(\mathbb{R}_{+}^{n}, \mathbb{R}_{+}^{m}\right)$. Then $\widetilde{T_{2}}(A) \in \pi\left(K_{1}, K_{2}\right)$. By Lemma 3.39, we know that $L$ is an onto preserver of $\pi\left(K_{1}, K_{2}\right)$, so that $L \widetilde{T_{2}}(A) \in \pi\left(K_{1}, K_{2}\right)$. Hence, $\widetilde{L_{1}}=\widetilde{T_{1}} L \widetilde{T_{2}}(A) \in \pi\left(\mathbb{R}_{+}^{n}, \mathbb{R}_{+}^{m}\right)$.

REMARK 3.41. It follows from the above result that when $L$ is an onto preserver of $S\left(K_{1}, K_{2}\right)$, the map $\widetilde{L}$ is also of the form $A \mapsto \widetilde{X} A \widetilde{Y}$, for some invertible matrices $\widetilde{X}$ and $\widetilde{Y}$. It can be easily seen that $\widetilde{X}=T_{1}^{t} X T_{2}$ and $\widetilde{Y}=S_{2}^{t} Y S_{1}$, which are nonnegative with respect to $\mathbb{R}_{+}^{m}$ and $\mathbb{R}_{+}^{n}$, respectively.

3.2.5. Preservers of left semipositivity. We end with the notion of left semipositivity and a preserver result concerning the same.

Definition 3.42. Let $A \in M_{m, n}$. We say $A$ is left $\left(K_{1}, K_{2}\right)$-semipositive if there exists $x \in K_{2}^{*}$ such that $A^{t} x \in\left(K_{1}^{*}\right)^{\circ}$.

The set of all left $\left(K_{1}, K_{2}\right)$-semipositive matrices will be denoted by $L S\left(K_{1}, K_{2}\right)$.

LEMmA 3.43. Let $K_{1}$ and $K_{2}$ be proper cones in $\mathbb{R}^{n}$ and $\mathbb{R}^{m}$, respectively. Then the following hold:

1. Let $Q_{1} \in \pi\left(K_{2}, \mathbb{R}_{+}^{m}\right)$ and $Q_{2} \in \pi\left(K_{1}, \mathbb{R}_{+}^{n}\right)$ be invertible. If $A \in L S\left(\mathbb{R}_{+}^{n}, \mathbb{R}_{+}^{m}\right)$, then $Q_{1}^{-1} A Q_{2} \in$ $\left.L S\left(K_{1}, K_{2}\right)\right)$.

2. Let $S_{1} \in \pi\left(\mathbb{R}_{+}^{m}, K_{2}\right)$ and $S_{2} \in \pi\left(\mathbb{R}_{+}^{n}, K_{1}\right)$ be invertible. If $\left.B \in L S\left(K_{1}, K_{2}\right)\right)$, then $S_{1}^{-1} B S_{2} \in$ $L S\left(\mathbb{R}_{+}^{n}, \mathbb{R}_{+}^{m}\right)$.

The following theorem can be proved and the proof follows similar to Lemma 3.2 of [9].

THEOREM 3.44. If $L: M_{m, n} \rightarrow M_{m, n}$ is an onto preserver of $S\left(K_{1}, K_{2}\right)$, then $L$ is also an onto preserver of $L S\left(K_{1}, K_{2}\right)$.

TheOREm 3.45. Let $S_{2} \in \pi\left(\mathbb{R}_{+}^{n}, K_{1}\right), Q_{2} \in \pi\left(K_{1}, \mathbb{R}_{+}^{n}\right), S_{1} \in \pi\left(\mathbb{R}_{+}^{m}, K_{2}\right)$, and $Q_{1} \in \pi\left(K_{2}, \mathbb{R}_{+}^{m}\right)$ be invertible matrices. Let $T_{1}(A)=Q_{1} A Q_{2}^{-1}$ and $T_{2}(A)=S_{1} A S_{2}^{-1}$. If $L: M_{m, n} \rightarrow M_{m, n}$ is an onto preserver of $S\left(K_{1}, K_{2}\right)$, then $L_{1}=T_{1} L T_{2}$ is an into preserver of $S\left(\mathbb{R}_{+}^{n}, \mathbb{R}_{+}^{m}\right)$ and $L_{1}^{-1}$ is an into preserver of $L S\left(\mathbb{R}_{+}^{n}, \mathbb{R}_{+}^{m}\right)$.

Proof. By Theorem 3.27, $L_{1}$ is an into preserver of $S\left(\mathbb{R}_{+}^{n}, \mathbb{R}_{+}^{m}\right)$. It can be easily seen that $L_{1}^{-1}$ is an into preserver of $L S\left(\mathbb{R}_{+}^{n}, \mathbb{R}_{+}^{m}\right)$.

\section{Appendix.}

\section{Proof of Theorem 3.10 - the $3 \times 3$ case}

Let $L$ be an invertible linear map on $M_{3}$ and let $A=\mathbf{x y}^{t}$ be a rank one matrix. Then, the matrix representation of $L(A)$ can be expressed as follows: Write $A=\mathbf{x y}^{t}$, where $\mathbf{x}=\left(x_{1}, x_{2}, x_{3}\right)^{t}$ and $\mathbf{y}=$ $\left(y_{1}, y_{2}, y_{3}\right)^{t}$. We then have 


$$
\begin{aligned}
& L(A)=\left[\begin{array}{lll}
l_{11} x_{1}+l_{14} x_{2}+l_{17} x_{3} & l_{21} x_{1}+l_{24} x_{2}+l_{27} x_{3} & l_{31} x_{1}+l_{34} x_{2}+l_{37} x_{3} \\
l_{41} x_{1}+l_{44} x_{2}+l_{47} x_{3} & l_{51} x_{1}+l_{54} x_{2}+l_{57} x_{3} & l_{61} x_{1}+l_{64} x_{2}+l_{67} x_{3} \\
l_{71} x_{1}+l_{74} x_{2}+l_{77} x_{3} & l_{81} x_{1}+l_{84} x_{2}+l_{87} x_{3} & l_{91} x_{1}+l_{94} x_{2}+l_{97} x_{3}
\end{array}\right] y_{1}+ \\
& {\left[\begin{array}{lll}
l_{12} x_{1}+l_{15} x_{2}+l_{18} x_{3} & l_{22} x_{1}+l_{25} x_{2}+l_{28} x_{3} & l_{32} x_{1}+l_{35} x_{2}+l_{38} x_{3} \\
l_{42} x_{1}+l_{45} x_{2}+l_{48} x_{3} & l_{52} x_{1}+l_{55} x_{2}+l_{58} x_{3} & l_{62} x_{1}+l_{65} x_{2}+l_{68} x_{3} \\
l_{72} x_{1}+l_{75} x_{2}+l_{78} x_{3} & l_{82} x_{1}+l_{85} x_{2}+l_{88} x_{3} & l_{92} x_{1}+l_{95} x_{2}+l_{98} x_{3}
\end{array}\right] y_{2}+} \\
& {\left[\begin{array}{lll}
l_{13} x_{1}+l_{16} x_{2}+l_{19} x_{3} & l_{23} x_{1}+l_{26} x_{2}+l_{29} x_{3} & l_{33} x_{1}+l_{36} x_{2}+l_{39} x_{3} \\
l_{43} x_{1}+l_{46} x_{2}+l_{49} x_{3} & l_{53} x_{1}+l_{56} x_{2}+l_{59} x_{3} & l_{63} x_{1}+l_{66} x_{2}+l_{69} x_{3} \\
l_{73} x_{1}+l_{76} x_{2}+l_{79} x_{3} & l_{83} x_{1}+l_{86} x_{2}+l_{89} x_{3} & l_{93} x_{1}+l_{96} x_{2}+l_{99} x_{3}
\end{array}\right] y_{3},} \\
& \text { where } l_{i j}, i=1, \ldots, 9, j=1, \ldots, 9 \text { are fixed real numbers. }
\end{aligned}
$$

Our aim is to prove that when $A_{1}$ is semipositive, $L\left(A_{1}\right)$ is mapped to a rank one (semipositive) matrix. The proof involves several steps. Here $A_{1}$ represents a rank one matrix of the form $A_{1}=\mathbf{x y}_{1}^{t}$, where $\mathbf{x}=\left(x_{1}, x_{2}, x_{3}\right)^{t}$ and $\mathbf{y}_{1}=\left(y_{1}, 0,0\right)^{t}$. We only indicate the main steps and include the proofs only when necessary. We shall have an occasion to use the following Theorem of the Alternative.

TheOREm 4.1 ([5, Theorem 2.8]). For an $m \times n$ matrix A, one and only one of the following alternatives holds.

(a) There exists $x \geq 0$ such that $A x>0$.

(b) There exists $0 \neq y \geq 0$ such that $-A^{t} y \geq 0$.

The first step is the following.

LEMma 4.2. Suppose $A_{1}$ is semipositive and $L$ is an invertible linear preserver of semipositivity. Then, the matrix $C_{1}=\left[\begin{array}{lll}l_{11} & l_{14} & l_{17} \\ l_{21} & l_{24} & l_{27} \\ l_{31} & l_{34} & l_{37}\end{array}\right]$ has rank one.

The proof is very similar to the one given in Theorem 3.9. One can also prove that the matrices $\left[\begin{array}{lll}l_{41} & l_{44} & l_{47} \\ l_{51} & l_{54} & l_{57} \\ l_{61} & l_{64} & l_{67}\end{array}\right]$ and $\left[\begin{array}{lll}l_{71} & l_{74} & l_{77} \\ l_{81} & l_{84} & l_{87} \\ l_{91} & l_{94} & l_{97}\end{array}\right]$ have rank one.

The second step is in proving that $L\left(A_{1}\right)$ contains a positive column when $A_{1}$ is semipositive. This is an important step in the proof. Let us denote by $P_{1}, P_{2}$, and $P_{3}$ the following numbers: $P_{1}:=l_{11} x_{1}+l_{14} x_{2}+$ $l_{17} x_{3}, P_{2}:=l_{41} x_{1}+l_{44} x_{2}+l_{47} x_{3}$, and $P_{3}:=l_{71} x_{1}+l_{74} x_{2}+l_{77} x_{3}$.

LEMMA 4.3. If $L$ is an invertible linear map on $M_{3}$ that preserves $S\left(\mathbb{R}_{+}^{3}\right)$, then $L\left(A_{1}\right)$ contains a positive column, when $A_{1}$ is semipositive.

Proof. Since $L\left(A_{1}\right)$ is redundantly semipositive, assume without loss of generality that the submatrix

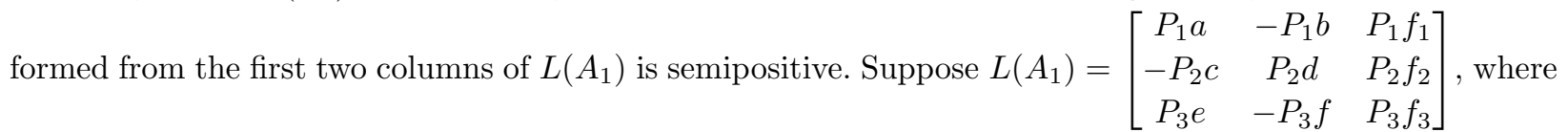
$a>0, d>0, e>0, b \geq 0, c \geq 0, f \geq 0$ and $f_{1}, f_{2}, f_{3} \in \mathbb{R}$. The proof involves the following steps.

Suppose $f_{3}>0$ : If $f_{1}>0, f_{2}>0$, then $L\left(A_{1}\right)$ has a positive column. The next steps involve verifying that the following subcases fail. In each such subcase, the idea is to find a semipositive matrix $B$ such that $L(B)$ is not semipositive. 
1. $f_{1}>0$ and $f_{2} \leq 0$.

2. $f_{1} \leq 0$ and $f_{2}>0$.

3. $f_{1} \leq 0$ and $f_{2} \leq 0$.

Suppose $f_{3} \leq 0$ : We discuss various subcases here too and check each such subcase fails. The argument is once again to find a semipositive matrix $B$ such that $L(B)$ is not semipositive.

1. $f_{1}>0$ and $f_{2}>0$.

2. $f_{1}>0$ and $f_{2} \leq 0$.

3. $f_{1} \leq 0$ and $f_{2}>0$.

4. $f_{1} \leq 0$ and $f_{2} \leq 0$.

This last step involves further subcases as follows: (i) $f_{3}<0$, (ii) $f_{3}=0$ and $f_{1}<0$, and finally (iii) $f_{3}=0, f_{1}=0$, and $f_{2}<0$. For completeness, we illustrate the proof of the following case: $f_{3}>0, f_{1} \leq 0$, and $f_{2} \leq 0$. We have

$$
L\left(A_{1}\right)=\left[\begin{array}{rrr}
P_{1} a & -P_{1} b & -P_{1} f_{1} \\
-P_{2} c & P_{2} d & -P_{2} f_{2} \\
P_{3} e & -P_{3} f & P_{3} f_{3}
\end{array}\right] \text {, where } a>0, d>0, e>0, b \geq 0, c \geq 0, f \geq 0, f_{1} \geq 0, f_{2} \geq 0, f_{3}>0 .
$$

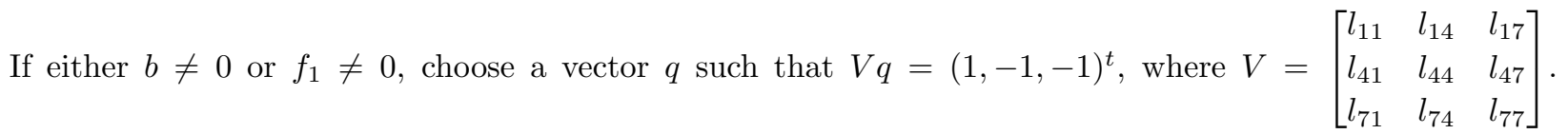
Form the semipositive matrix $B$ as follows: $B=\left[\begin{array}{lll}q_{1} & 0 & 0 \\ q_{2} & 0 & 0 \\ q_{3} & 0 & 0\end{array}\right]+y_{2}\left[\begin{array}{lll}0 & x_{1} & 0 \\ 0 & x_{2} & 0 \\ 0 & x_{3} & 0\end{array}\right]$, where $y_{2}>0$. Then, $L(B)=\left[\begin{array}{rrr}a & -b & -f_{1} \\ c & -d & f_{2} \\ -e & f & -f_{3}\end{array}\right]+y_{2} L\left(\left[\begin{array}{lll}0 & x_{1} & 0 \\ 0 & x_{2} & 0 \\ 0 & x_{3} & 0\end{array}\right]\right)$. Choose a $y_{2}$ small enough so that $B$ is semipositive, whereas $L(B)$ is not semipositive. Suppose both $b$ and $f_{1}$ are zero. If there is no $y_{2}>0$ such that $L(B)$ is not semipositive, then form the matrix $B_{1}=\left[\begin{array}{lll}q_{1} & 0 & 0 \\ q_{2} & 0 & 0 \\ q_{3} & 0 & 0\end{array}\right]+\left(-y_{2}\right)\left[\begin{array}{lll}0 & x_{1} & 0 \\ 0 & x_{2} & 0 \\ 0 & x_{3} & 0\end{array}\right]+y_{3}\left[\begin{array}{lll}0 & 0 & x_{1} \\ 0 & 0 & x_{2} \\ 0 & 0 & x_{3}\end{array}\right]$, where $y_{2}>0$ is small enough and $y_{3}>0$. Notice that $B_{1}$ is a semipositive matrix. Now choose $y_{3}$ sufficiently small to make $L\left(B_{1}\right)$ not semipositive. Combining all the steps mentioned above, we prove that $L\left(A_{1}\right)$ has a positive column, when $L$ preserves semipositivity.

A similar argument will ensure that $L\left(A_{2}\right)$ and $L\left(A_{3}\right)$ also have positive columns when $L$ preserves semipositive matrices and the matrices $A_{2}$ and $A_{3}$ are semipositive.

We thus have $L\left(A_{1}\right)=\left[\begin{array}{lll}P_{1} & \alpha_{1} P_{1} & \beta_{1} P_{1} \\ P_{2} & \alpha_{2} P_{2} & \beta_{2} P_{2} \\ P_{3} & \alpha_{3} P_{3} & \beta_{3} P_{3}\end{array}\right]$ for some $\alpha_{i}$ and $\beta_{i} \in \mathbb{R}$ for $i=1,2,3$.

Before proceeding further, let us denote by $E$ the matrix $\left[\begin{array}{ll}\alpha_{1} & \beta_{1} \\ \alpha_{2} & \beta_{2} \\ \alpha_{3} & \beta_{3}\end{array}\right]$.

The next step is the following result. 
Lemma 4.4. Let $L$ be an invertible linear map on $M_{3}$ that preserves semipositive matrices. Assume that $A_{1}$ is semipositive, the first column of $L\left(A_{1}\right)$ is positive, and the matrix $E$ is semipositive. Then, $L\left(A_{1}\right)$ has rank one.

Proof. The first step is in proving that the matrix $E$ cannot contain any $2 \times 2$ submatrix that is minimally semipositive. The second step is in proving that both the columns of $E$ cannot be positive. Consider the following flowchart.

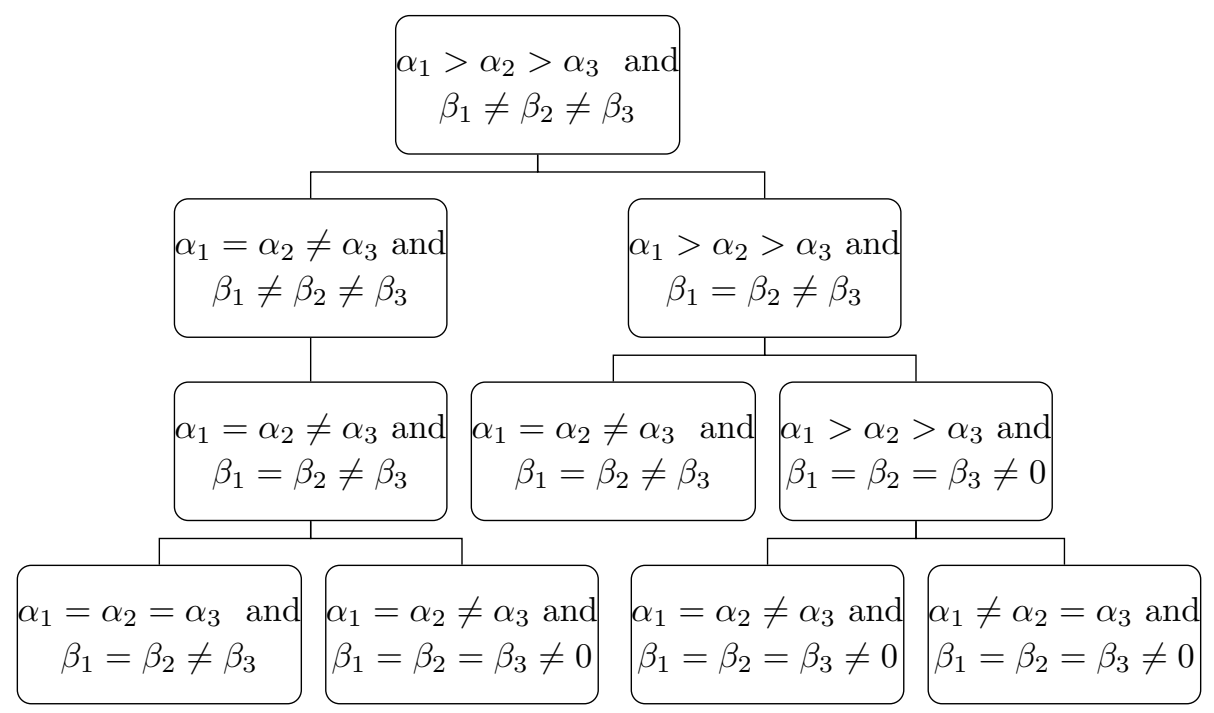

The remaining argument of Lemma 4.4 is in proving that each of the steps in the above flowchart fails. As in the previous lemma, the proof involves finding a semipositive matrix $B$ such that $L(B)$ is not semipositive. We include the proof of the following two cases alone.

Suppose $\alpha_{1}>\alpha_{2}>\alpha_{3}$ and $\beta_{1} \neq \beta_{2} \neq \beta_{3}$.

Suppose the vectors $\left(\alpha_{1}, \alpha_{2}, \alpha_{3}\right)^{t}$ and $\left(-\beta_{1},-\beta_{2},-\beta_{3}\right)^{t}$ are linearly dependent. Let $\delta>0$ be such that $\left(-\beta_{1},-\beta_{2},-\beta_{3}\right)^{t}=-\delta\left(\alpha_{1}, \alpha_{2}, \alpha_{3}\right)^{t}$. Choose a vector $q$ so that $V q=(1,-1,1)^{t}$ and form a semipositive matrix $B$ as before. Observe that $L(B)=\left[\begin{array}{rrr}1 & \alpha_{1} & -\delta \alpha_{1} \\ -1 & -\alpha_{2} & \delta \alpha_{2} \\ 1 & \alpha_{3} & -\delta \alpha_{3}\end{array}\right]+y_{2} L\left(\left[\begin{array}{lll}0 & x_{1} & 0 \\ 0 & x_{2} & 0 \\ 0 & x_{3} & 0\end{array}\right]\right)$. If there exists a $y_{2}>0$ small enough so that $L(B)$ is not semipositive, then we get a contradiction to our assumption. Else, choose a vector $q$ such that $V q=(1,1,-1)^{t}$ and proceed as above. Notice that in this case, it is possible to choose a $y_{2}$ small enough that makes $L(B)$ not semipositive in at least one of the cases.

Suppose the vectors $\left(\alpha_{1}, \alpha_{2}, \alpha_{3}\right)^{t}$ and $\left(-\beta_{1},-\beta_{2},-\beta_{3}\right)^{t}$ are linearly independent. Consider the invertible ma$\operatorname{trix} D=\left[\begin{array}{rrr}-1 & -\alpha_{1} & \beta_{1} \\ 1 & \alpha_{2} & -\beta_{2} \\ -1 & -\alpha_{3} & \beta_{3}\end{array}\right]$. Suppose $\operatorname{det} D<0$. Notice that $D^{-1}=\left(\frac{1}{\operatorname{det} D}\right)\left[\begin{array}{ccc}* & * & * \\ * & * & * \\ \alpha_{2}-\alpha_{3} & \alpha_{1}-\alpha_{3} & \alpha_{1}-\alpha_{2}\end{array}\right]$. Notice that the last row entries of the matrix are positive. Therefore, $u=\left(\frac{1}{\operatorname{det} D}\right)\left(\alpha_{2}-\alpha_{3}, \alpha_{1}-\alpha_{3}, \alpha_{1}-\right.$ $\left.\alpha_{2}\right)^{t}<0$. Now $D^{t} u=-D^{t}(-u)=(0,0,1)^{t} \geq 0$. Thus, $D$ is not semipositive (by the Theorem of the Alternative 4.1). Now choose a vector $q$ so that $V q=(-1,1,-1)^{t}$ and form the semipositive matrix 
$B$ as in the previous steps. Then, $L(B)=\left[\begin{array}{rrr}-1 & -\alpha_{1} & \beta_{1} \\ 1 & \alpha_{2} & -\beta_{2} \\ -1 & -\alpha_{3} & \beta_{3}\end{array}\right]+y_{2} L\left(\left[\begin{array}{lll}0 & x_{1} & 0 \\ 0 & x_{2} & 0 \\ 0 & x_{3} & 0\end{array}\right]\right)$. It is possible to choose a $y_{2}$ small enough so that $L(B)$ is not semipositive. If $\operatorname{det} D>0$, then it is easy to check that $(-D)^{-1}=\left(\frac{1}{-\operatorname{det} D}\right)\left[\begin{array}{ccc}* & * & * \\ * & * & * \\ \alpha_{2}-\alpha_{3} & \alpha_{1}-\alpha_{3} & \alpha_{1}-\alpha_{2}\end{array}\right]$. The vector $u=\left(\frac{1}{-\operatorname{det} D}\right)\left(\alpha_{2}-\alpha_{3}, \alpha_{1}-\alpha_{3}, \alpha_{1}-\alpha_{2}\right)^{t}<0$ and $-\left(-D^{t}\right)(-u)=(0,0,1)^{t} \geq 0$. Once again, by the Theorem of the Alternative 4.1 , we have $-D \notin S\left(\mathbb{R}_{+}^{3}\right)$. Now choose a vector $q$ so that $V q=(1,-1,1)^{t}$ and proceed as in the case when $\operatorname{det} D<0$.

Combining all the steps in the above flowchart, we see that $L\left(A_{1}\right)=\left[\begin{array}{lll}P_{1} & \alpha P_{1} & -\beta P_{1} \\ P_{2} & \alpha P_{2} & -\beta P_{2} \\ P_{3} & \alpha P_{3} & -\beta P_{3}\end{array}\right]$, where $\alpha>0$ and $\beta \geq 0$.

The remaining steps involve the following lemmas.

Lemma 4.5. Let $L$ be an invertible linear map on $M_{3}$ that preserves semipositive matrices. Then, the following hold.

1. Suppose the first column of $L\left(A_{1}\right)$ is positive, $-E$ is semipositive, whereas $E$ is not. Then, $L\left(A_{1}\right)$ has rank one.

2. Suppose the first column of $L\left(A_{1}\right)$ is positive and both $E$ and $-E$ are not semipositive. Then, $L\left(A_{1}\right)$ has rank one.

A similar argument shows that $L\left(A_{2}\right)$ and $L\left(A_{3}\right)$ have rank one. We now have the result on the structure of a preserver in this case. The proof is similar to that of Theorem 3.11.

TheOREm 4.6. Let $L$ be an invertible linear map on $M_{3}$ that preserves $S\left(\mathbb{R}_{+}^{3}\right)$. Then $L(A)=X A Y$ for all $A \in M_{3}$, where $X$ is a row positive matrix and $Y$ is an inverse nonnegative matrix.

Acknowledgements. The authors thank the anonymous referee for a critical reading of the manuscript and for numerous suggestions that have helped us improve the presentation tremendously. The authors also thank Professors C. R. Johnson, K.C. Sivakumar, and M. J. Tsatsomeros for their suggestions, comments, and encouragement. The second author acknowledges the Council of Scientific and Industrial Research (CSIR), India, for support in the form of Junior and Senior Research Fellowships (Award No. 09/997(0033)/2015EMR-I).

\section{REFERENCES}

[1] A. Chandrashekaran, S. Jayaraman, and V.N. Mer. Semipositivity of linear maps relative to proper cones in finite dimensional real Hilbert spaces. Electron. J. Linear Algebra, 34:304-319, 2018.

[2] A. Chandrashekaran, S. Jayaraman, and V.N. Mer. A characterization of nonnegativity relative to proper cones. Indian J. Pure Appl. Math., 51:935-944, 2020.

[3] G.P. Barker. Theory of cones. Linear Algebra Appl., 39:63-91, 1981.

[4] A. Berman and R.J. Plemmons. Nonnegative Matrices in the Mathematical Sciences. SIAM Classics in Applied Mathematics, Philadelphia, 1994.

[5] B. Cain, D. Hershkowitz, and H. Schneider. Theorems of the alternative for cones and Lyapunov regularity of matrices. Czechoslovak Math. J., 47:487-499, 1997. 
[6] P.N. Choudhury, R.M. Kannan, and K.C. Sivakumar. New contributions to semipositive and minimally semipositive matrices. Electron. J. Linear Algebra, 34:35-53, 2018.

[7] P.N. Choudhury, R.M. Kannan, and K.C. Sivakumar. A note on linear preservers of semipositive and minimally semipositive matrices. Electron. J. Linear Algebra, 34:687-694, 2018.

[8] J. Dieudonnè. Sur unè gènèralisation du groupe orthogonal à quatre variables [On a generalization that an orthogonal group has four variables]. Archiv der Mathematik, 1:282-287, 1948/1949.

[9] J. Dorsey, T. Gannon, N. Jacobson, C.R. Johnson, and M. Turnansky. Linear preservers of semi-positive matrices. Linear Multilinear Algebra, 64:1853-1862, 2016.

[10] E. Haynsworth, M. Fiedler, and V. Ptak. Extreme operators on polyhedral cones. Linear Algebra Appl., 13:163-172, 1976.

[11] C.R. Johnson. Sign patterns of inverse non-negative matrices. Linear Algebra Appl., 55:69-80, 1983.

[12] C.R. Johnson, M.K. Kerr, and D.P. Stanford. Semipositivity of matrices. Linear Multilinear Algebra, 37:265-271, 1994.

[13] C. Lautemann. Linear transformations on matrices: rank preservers and determinant preservers (Note). Linear Multilinear Algebra, 10:343-345, 1981.

[14] C-K. Li and S. Pierce. Linear preserver problems. Amer. Math. Monthly, (August-September), 591-605, 2001.

[15] M. Marcus and B.N. Moyls. Transformations on tensor product spaces. Pacific J. Math., 9:1215-1221, 1959.

[16] M. Marcus and B.N. Moyls. Linear transformations on algebras of matrices. Canad. J. Math., 11:61-66, 1959.

[17] H. Minc. Linear transformations on nonnegative matrices. Linear Algebra Appl., 9:149-153, 1974.

[18] L. Rodman and P. Semrl. A localization technique for linear preserver problems. Linear Algebra Appl., 433:2257-2268, 2010.

[19] M.J. Tsatsomeros. Geometric mapping properties of semipositive matrices. Linear Algebra Appl., 498:349-359, 2016.

[20] X. Zhang, Z. Tang, and C. Cao. Preserver Problems on Spaces of Matrices. Science Press, Beijing, 2006. 